\title{
EFFECTS OF ARTIFICIAL TEMPERATURE REGULATION ON SPLENIC NODULAR TISSUE IN THE EXTERIORIZED RAT SPLEEN: AN APPLICATION OF BIOENGINEERING PRINCIPLES*†
}

\author{
Edward C. Pliske \\ Department of Anatomy \\ University of Michigan School of Medicine \\ Ann Arbor, Michigan 48104
}

\section{INTRODUCTION}

Even to the casual observer, it has become axiomatic that all animals, although comprising two distinct groups, carry out their normal metabolic and physiologic functions through a wide range of environmental temperature. A wareness of this concept unquestionably dates back to ancient man. One group (poikilotherms), exhibits a remarkable parallelism between body temperature, inclusive of organs, with that of the ambient or environmental temperature. The other group (homeotherms), is characterized by an ability to generate and maintain a consistent body temperature irrespective of fluctuations that occur in environmental temperature.

In general, homeothermic animals, including laboratory rodents, when subjected to an environmental temperature depression (general hypothermia) or an elevation (general hyperthermia), react to reestablish their body temperature norm. Allowing for species differencies, the type and rate of reaction varies according to the intensity and duration of the thermal stimulus, but invariably results in the activation of some or all of established temperature-regulatory mechanisms within the animal. These include the central and autonomic nervous systems, endocrine and exocrine glands, skin, cardiovascular system, and fluid depots (including blood). The regulatory mechanisms appear to be competent only within critical levels of environmental variation, beyond which body temperature progressively deteriorates in favor of an overwhelming ambient temperature. The composite of these subjects has been extensively reviewed by Herzfeld. ${ }^{23}$

Students of comparative physiology have long utilized a temperature coefficient, or $\mathrm{Q}_{10}$, to measure the rate of metabolic reaction and tissue response in poikilotherms, based on a $10^{\circ} \mathrm{C}$ decrease or increase in environmental temperature. The temperature coefficient was more useful with poikilotherms than with homeotherms until the advent of artificial temperature regulation as a technique or method. Attempts to regulate artificially the temperature of a single organ or a selected region of tissues of the homeotherm body are generally known as local hypothermia or hyperthermia, as the case may be. Within poorly defined limits, extreme local hypothermia tends to isolate a homeotherm organ from the influence of temperature-regulatory mechanisms when normal body temperature is induced to remain constant. In such instances, homeotherm organs approach a temperature-related circumstance that is natural to poikil-

*Submitted August 27, 1971; accepted January 3, 1972.

†This work was supported in part by NSF Institutional grant No. 30 from The Horace H. Rackham School of Graduate Studies and NIH medical project No. 15 grant, University of Michigan Medical School. 
otherms and in which $\mathrm{O}_{2}$ requirements and metabolic functions tend to decrease concomitantly with a decrease in temperature.

The science of cryobiology, in which entire organisms, organs, tissues, and cells reveal a high metabolic tolerance to wide extremes of temperature depression, is well known. In contrast, temperature elevations of less than one $Q_{10}$ above body temperature are equally known to result in tissue and organ damage to the homethermic organism.9,36 It therefore follows that the effective experimental range of local hypothermia is considerably more extensive than would be true for local hyperthermia. The methods or techniques by which local hypothermia are produced vary widely. In some instances the effects of cold analgesia are sought, in others, a reduction in metabolic rate or control of hemorrhage. Based on the foregoing, it is understandable that local hypothermia has found and continues to find broad clinical applications. Selected examples of these are as follows: Wangensteen and associates ${ }^{41}$ treated patients suffering upper gastrointestinal bleeding by means of local gastric cooling. A resultant marked reduction in gastric blood flow as well as in gastric secretion was observed to occur. The analgesic effects of cold on local tissues in ischemic limbs and intestines have been reported by Allen $^{1}$ and also by Crossman and colleagues $^{7}$ as an adjunct to reduction of pain in amputations. Local modifications of hypothermia have been shown to be of especial value in the prevention of ischemia in brain surgery ${ }^{11}$ and in the prevention of anoxia in intracardiac surgery. ${ }^{46}$ Means of achieving the cryogenic effect in local tissues include intragastric balloons, $4,21,27,29,42$ extracorporeal cooling of the blood stream, ${ }^{8,33}$ exteriorization of an organ in a Ringer's bath solution, ${ }^{30}$ visceral cooling, ${ }^{5}$ and cooling coils. ${ }^{6}$ The induction of true local hypothermia under controlled conditions has been rarely accomplished, because of an array of technical problems that vary according to the method employed.

In the present study an attempt has been made to determine the histological changes that occur in the living exteriorized rat spleen when its temperature is artifically regulated. Particular attention has been given to temperature-induced changes that modify the nodular lymphoid parenchyma at selected levels of local hypothermia. The surgical accessibility of the rat spleen and the relative ease with which it can be exteriorized makes it an organ uniquely suitable for this type of study. A thermal regulatory apparatus, designed for the study, is also described.

\section{MATERIALS AND METHODS \\ The Thermal Regulatory Apparatus}

A schema of the structural components, electronic circuitry, and fluid circuitry comprising the thermal regulatory apparatus used in this study is illustrated in FIGURE 1. Many of the components were designed and constructed by the author; others were purchased and adapted for use. The apparatus consisted of six separate but functionally related and interdependent units. These were: Unit I (cold-exchanger complex), Unit II (heat-exchanger complex), Unit III (telethermometer and probes), Unit IV (thermistemp temperature controller), Unit V (heated animal table), and Unit VI (the thermal end capsule).

Unit I consisted of the cold exchanger (CE), its filling chamber (FC), and its bivent valve (V4), together with trivent valves (VI and $V 2$ ) and bypass valve (V3). A glass air-bleeding manometer (A) served as a water level gauge and as an 


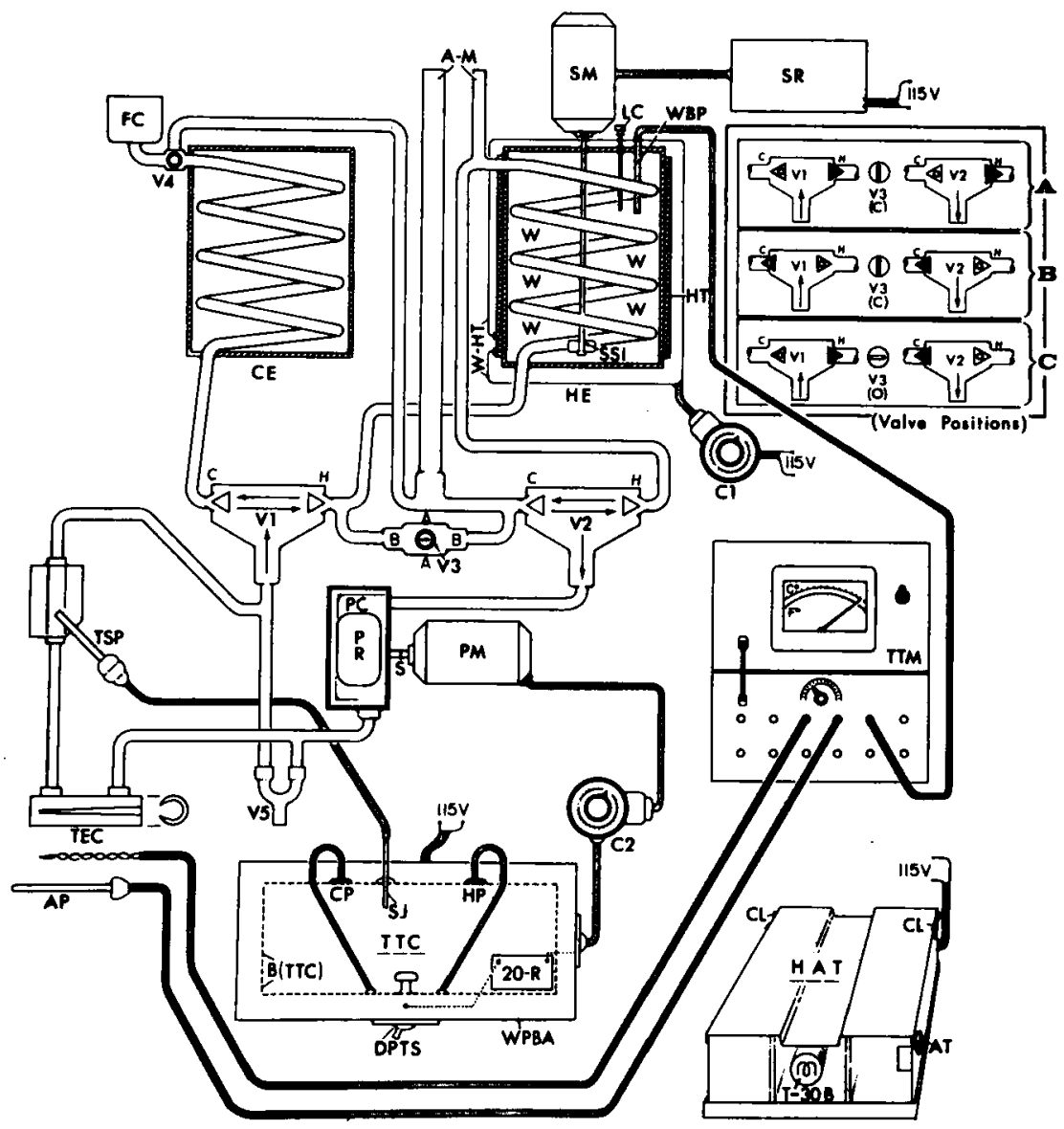

FIGURE 1. Schema of the thermal regulatory apparatus. Key: AM-air-bleeding manometers; AP-anal probe; AT-adjustable thermostat; B(TTC)-base of thermister temperature controller; $\mathrm{C}$-cold; $\mathrm{C}$ 1-powerstat control to heating tape; $\mathrm{C} 2$-powerstat rate control to pump motor; $\mathrm{CE}$-cold exchanger; $\mathrm{CL}$-two of four animal-securing cleats; $\mathrm{CP}$-cold plug of thermistemp temperature controller; DPTS-double-pole, double-throw toggle switch; FC-filling chamber; H-hot; HE-heat exchanger; HP-hot plug of thermistemp temperature controller; HT-heating tape; HAT-sectional view heated animal table; LC-limit control; PC-pump chamber; PM-pump motor; PR-pump rotor; 2OR-20-ampere capacity relay; S-pump shaft; SJ-sensor jack of thermistemp temperature controller; SM-stirring motor; SR-stabilized resistance (rate control) to stirring motor; SSI-stirring shaft and impeller; TEC-thermal end capsule; TSP-temperature sensory probe of circulant; TTC-(Yellow Springs) thermistemp temperature controller; TTM-(Yellow Springs) 12-channel telethermometer; T-30B-tubular (30-watt) heating bulb; V 1-three-portal valve; $V$ 2-three-portal valve; V 3-by-pass valve ( $A A=$ open, $B B=$ closed); V 4-petcock valve; V 5-pressure clamps on rubber tubing of each arm of "Y"; W-water; WBP-water-bath probe; WHT-lead wires to heating tape; WPBA-wood platform base for thermistemp. 
air-bleeder. A separate compartment of the unit contained the pump motor (PM) arid pump ensemble (PC, PR, and S).

The cold exchanger was essentially a watertight, galvanized sheet metal tank (12 in. $X 10$ in. $X 6$ in.) with a concave floor. A drain tube was located at the lowest point of the concavity. A flat coil of $14 \mathrm{ft}$. of copper tubing (1/4 in. inside diameter) formed a second floor 2 in. above the lowest point of the sheet-metal floor. The copper tubing was overlain with 1/4-in.-mesh, galvanized hardware cloth. Points of exit and entry of the copper tube were soldered to the tank. The cold exchanger was designed for use with ice, dry ice, or prechilled cannisters of coolant.

The filling chamber, a sheet-metal tank (600.0-ml capacity) with drain and valve (V4), served to fill the entire apparatus with the fluid cryogen. Release of pressure clamps on valve 5 drained the apparatus. All tubing external to the cold exchanger, with the exception of the glass air-bleeder, was Tygon tubing (1/4 in. I.D.). All of the valves were of brass construction, with the exception of V5, which was rubber tubing. External surfaces of the cold exchanger were insulated with 1-in.-thick styrofoam. All valves and tubing external to the cold exchanger were wrapped with heavy aluminum foil.

The pump ensemble consisted of a 3-ampere/115-v AC series wound motor (PM), a pump housing of 1 -in. thick plexiglass, and a six-vane bronze rotor (PR). The volume of the pump chamber (PC) was $37.0 \mathrm{ml}$. A segment of firm rubber tube served as a universal joint between the motor shaft and the pump shaft (S). The design of the pump chamber (PC) and the rotor (PR) permitted an even flow from the pump rather than a pulsating one.

Unit II was contained in a round copper tank ( 6 in. $X 6-1 / 2$ in.). It contained a helix (12 ft of 1/4-in. copper tubing) soldered at points of entry into and exit from the tank shell. Stirring motor (SM), its shaft, and its impeller (SSI) equalized the heated water (W). Resistance stabilizer (SR) controlled the stirring rate to $70 \mathrm{rpm}$. Flexible heating tape (HT) (96 in. $X 1 \mathrm{in./768} \mathrm{watts)} \mathrm{was} \mathrm{applied}$ spirally to the external surface of the tank. Lead wires (W-HT) to powerstat (CI) permitted heat control of the water bath. Limit control switch (LC) provided for preset heat cutoff.

Unit III, a self-powered 12-channel telethermometer (TTM), Yellow Springs Instrument Co., recorded all required temperatures for the animals and for the operation of the apparatus. Stainless steel probe (WBP) was used to regulate the limit control switch and the rate of heating. An anal probe (AP) recorded body (core) temperature of the rat. A flexible, plastic-coated probe (above AP, FIG. 1) was inserted between the spleen and the internal surface of the thermal end capsule (TEC) to record temperature transfer.

Unit IV, a modified thermistemp temperature controller (TTC), Yellow Springs Instrument Co., Model 71, automatically controlled temperature delivery to the thermal end capsule by means of a probe (TSP) plugged into the sensor jack of the temperature controller. The latter, equipped with a cold plug (CP) and a hot plug (HP), was wired to a double-pole, double-throw switch (DPTS) located in the wooden platform base (WPBA) on which the temperature controller rested. A 20-ampere relay (20-R) installed between the switch and powerstat (C2) controlled the pump speed and resulting rate of fluid cryogen circulation.

Fluid circuitry. Directional arrows (FIG. 1) in the vertical stem of valves V1 and $V 2$ indicate afferent and efferent flow with respect to the pump chamber (PC). Horizontal arrows in the same valves indicate possibilities of flow with regard to the cold exchanger (vent c) or heat exchanger (vent $h$ ). Bypass valve 
(V3) was open (BB) or closed (AA) as required. The inset diagram of (valve positions) at the right of FIGURE 1 lettered $A, B$, and $C$ depict valve positions for each use of the apparatus: $A=$ cold exchanger alone, $B=$ heat exchanger alone, and $\mathrm{C}=$ cold and heat exchangers in combination.

Unit $V$, the heated animal table (HAT) $(6$ in. $X 9$ in. $X 3$ in.). was of plywood construction except for the top, which was 20-guage duraluminum. A trough extending through the top served to center the animal and to increase the warming area. Below the trough, a 30-watt tubular bulb (T-30B) provided heat for the axial area of the rat. Vents were located at either end of the trough. The two chambers lateral to the central heated chamber were not vented. Uniform heat was maintained by an adjustable thermostat (AT). Four cleats (CL) served to secure the animal.

Unit VI was constructed from burnished duraluminum rod (11/16 in. diameter $X 2$ in.). From one end, an off-center hole $12 \mathrm{~mm}$ in diameter was drilled so that the direction of the bore paralleled the length of the rod for $17 / 8$ in., thus leaving the final $1 / 8 \mathrm{in}$. undrilled. At its nearest point, the margin of the hole came to $1 / 32$ in. of breaking through the external surface of the rod. The thin remaining wall was then sawed through for the remaining depth of the bore. The margins of the cleft were widened to $1 / 8$ in. and dressed smooth by filing and use of fine grit. A crescent-shaped water passageway was milled into the thicker area of the rod wall for the entire extent of the $12-\mathrm{mm}$. bore. Inlet and outlet tubes were threaded into the water passageway, and the end of the rod from which the milling began was capped and sealed. Tests for uniformity in temperature transfer were made, and these resulted in minor modifications of the design of the water passageways. The design of the thermal end capsule (TEC) permitted easy insertion of the spleen into the $12-\mathrm{mm}$ bore and its mesentery in the slot. Removal of the organ from TEC was no more difficult. A pledget of saline-saturated cotton was placed in the blind end of the bore prior to insertion of the spleen. Another pledget closed the open end. Saline-saturated packs protected the margins of the incision and the minimally exposed splenic mesentery. An adjustable Fisher Clamp positioned and supported the thermal end capsule and contained spleen for the period of artificial temperature exposure. At the conclusion of an exposure the spleen was dislodged from the TEC with saline-wet swabs.

\section{EXPERIMENTAL ANIMALS AND CONTROLS}

Forty-eight female Sprague-Dawley rats of $160-170$ grams b/w. were used in this study. Sodium phenobarbital anesthesia was administered subcutaneously in two doses of $32.0 \mathrm{mg}$ each, an hour apart. The volume $/ \mathrm{mg}$ ratio was adjusted so that $0.1 \mathrm{ml}$ was equal to $5.0 \mathrm{mg} \mathrm{Na}$ phenobarbital. Animals were routinely anesthetized in pairs to allow selection of the one that responded most favorably to the anesthetic. A satisfactory level of anesthesia persisted for 6 to 8 hours.

Two groups of experimental animals were used. Group I consisted of 16 animals the spleens of which were exposed for three hours to $37^{\circ}, 30^{\circ}, 26^{\circ}$, and $15^{\circ}$. C. Splenectomy was performed immediately following the artificial temperature exposure. In Group II the spleens of 24 animals were exposed for three hours to $37^{\circ}, 30^{\circ}, 26^{\circ}, 20^{\circ}, 18^{\circ}$, and $14^{\circ} \mathrm{C}$. For this group, the spleens of all animals were returned to the body cavity for a period of 72 hours following artificial temperature exposure before splenectomy was performed. Two animals were exposed to each of the temperatures indicated for both Group I and Group II. The temperature controller was operated manually to depress splenic 
temperatures to a predetermined level. This required approximately five minutes. The temperature controller (TTC) automatically maintained the selected temperature within $\pm 0.1^{\circ} \mathrm{C}$ for the remainder of the three-hour exposure.

In order to determine the direct effect of thermal regulation on the spleens as distinguished from mechanical or manipulatory effects, eight controls were studied, two for each of the following: (1) adverse effects from insertion of the spleen into the thermal end capsule as well as its removal, (2) adverse effects from minimal drying, (3) the importance of maintaining total body temperature, and (4) the influence of minimal ether anesthesia in addition to $\mathrm{Na}$ phenobarbital. (1) and (2) were found to be insignificant, whereas (3) appeared to be very important both for survival of the animal and for the reliability of tissue results and (4) indicated no adverse effects.

\section{TISSUES}

A $10 \%$ unbuffered solution of formalin was used routinely as the fixative. Paraffin sections were cut serially at 8 and 10 micra. Pappenheim's modification of methyl green pyronin (as described by Slider and Downey) was used as the routine staining procedure. For comparison, a few slides from each spleen were stained with Harris hematoxylin and eosin and with Verhoeff's elastic stain.

\section{OBSERVATIONS}

\section{Group I (Three Hours of Artificially Regulated Temperature Followed by Splenectomy)}

Group $I 37^{\circ} \mathrm{C}$. The general size and organization of the nodular tissue and white pulp areas showed little change from that which might be expected from one normal spleen to another (FIG. 3). More critical observation, however, revealed a marked accentuation of the various regions that comprise a typical nodule. The germinal center in FIGURE 3 is large and clearly demarcated from the mantle zone of small lymphocytes except for the antiarterial sector. In the latter, the mantle zone has become thin and contains relatively few small lymphocytes. Progressing from this thin point along the mantle zone-germinal center junction on either side, it will be noted, irregular aggregates of mantle-zone lymphocytes project into the periphery of the germinal center and partially occupy anastomosing channel-like spaces. The squared area in FIGURE 3 is represented at higher magnification in FIGURE 4. At the extreme right of FIGURE 4 a

\begin{tabular}{|ll|}
\hline \multicolumn{2}{|c|}{ ABBREVIATIONS FOR FIGURES 2-26 } \\
A \& B.V.-artery & M.LC.-medium-sized lymphocy tes \\
C.C.-condensed cord of lymphocytes & MPG.-macrophage \\
C.H.-channel & M.S.-marginal sinus \\
D.P.-dark pole & M.Z.-marginal zone \\
G.C.-germinal center & R.A.-rarefied area \\
HC.BL.-hemocy toblast & RBC'S.-red blood corpuscles \\
H.S.L.-hyperchromatic small lymphocytes & RP. \& S.-red pulp; sinus \\
L.P.-light pole & RPHC.-red pulp hemopoietic center \\
L.R.-linear regeneration & S.LC.-small lymphocytes \\
MA.Z.-mantle zone & T.MPG.-tingible body macrophage \\
MET.-marginal metalophil & W.P.- white pulp \\
\hline
\end{tabular}




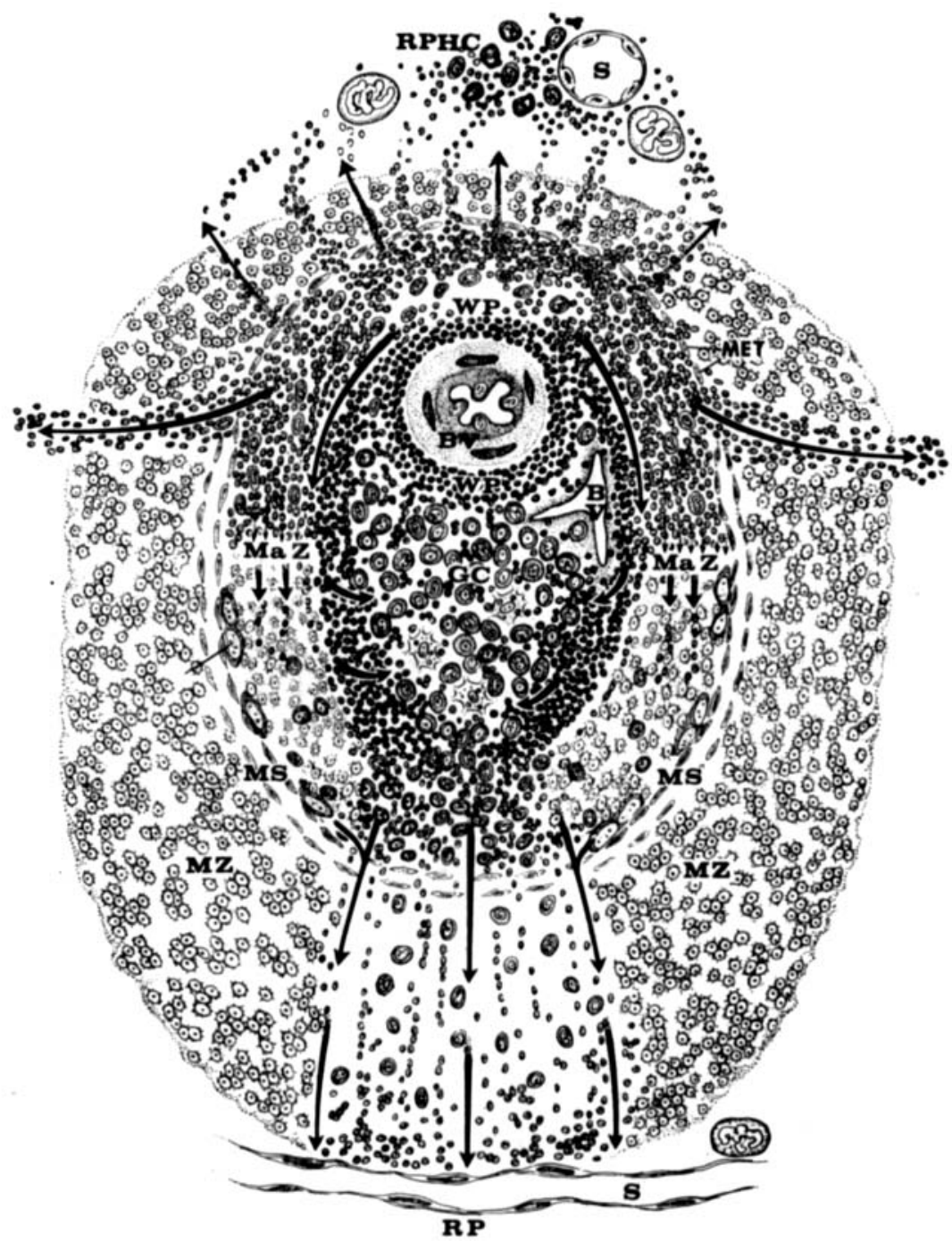

FIGURE 2. Schema of the most active points of release of cells from a hypothermic splenic nodule (see arrows in the marginal zone, M Z). Arrows in the lower part of the figure represent the antiarterial sector of the nodule in which cell release predominates. Arrows in the upper (nonsinus) area reveal a more diffuse release of cells between the nonhypertrophied metalophils (MET). The metalophils in the lower part of the Figure are represented in their hypertrophied state (MET). Arrows from the white pulp (WP) indicate the progression of small lymphocy tes in to the mantle zone $(\mathrm{M} \mathrm{Z})$, the germinal center, and the antiarterial sector of the nodule. RPHC, RP, and S represent red-pulp hemopoietic centers, red pulp, and red-pulp sinuses, respectively. 


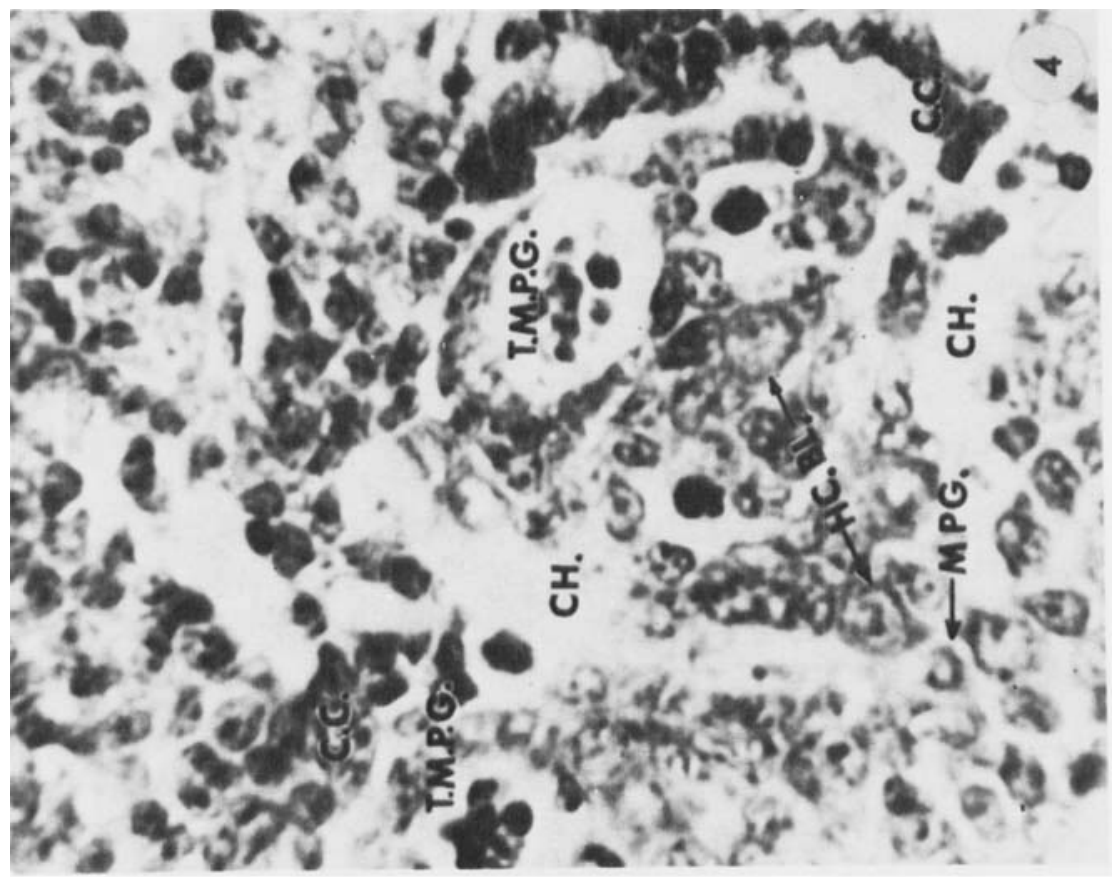

5.6.

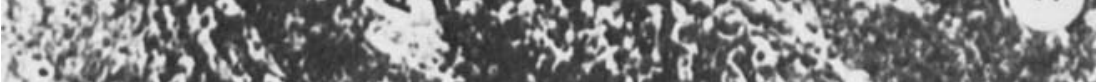

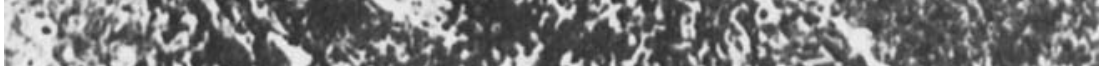

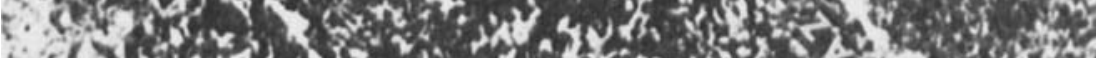

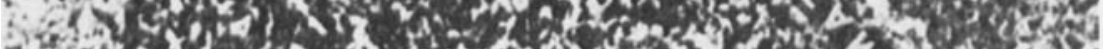
i. $x^{2}$.

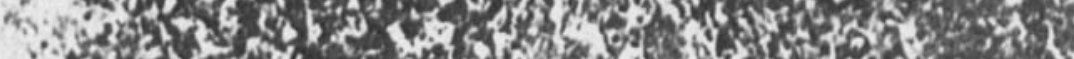

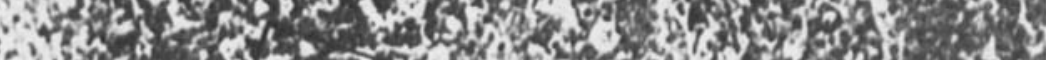

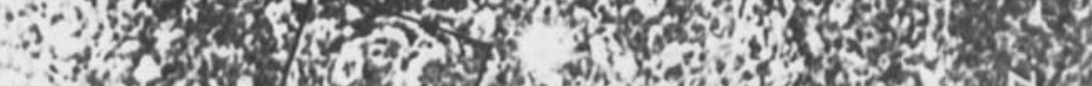

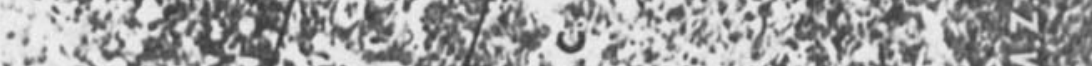

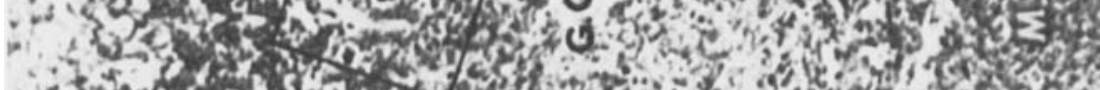

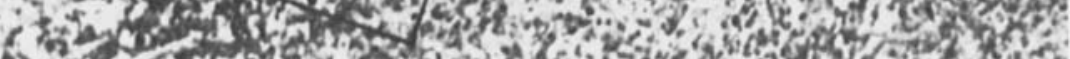

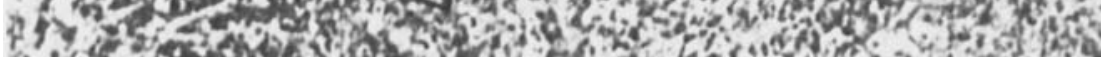

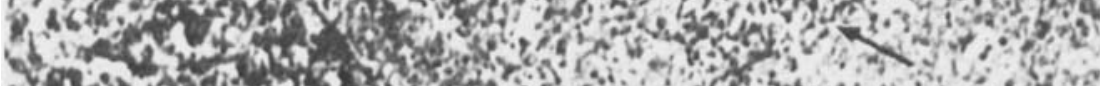

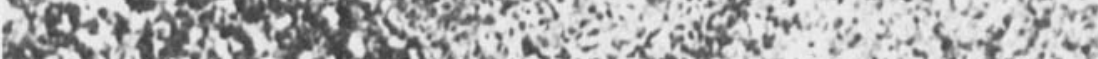

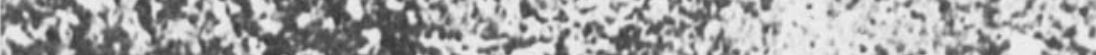

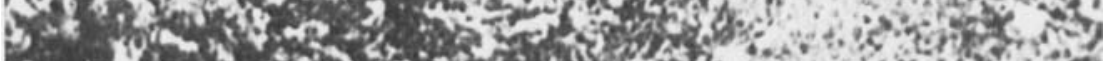

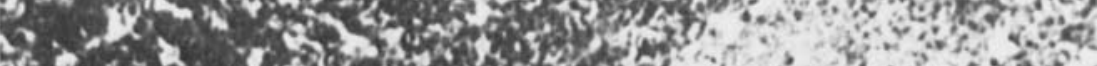

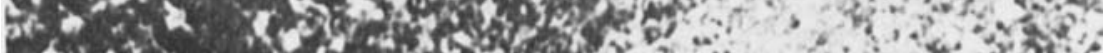

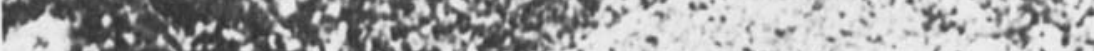
$\therefore$ ar V. 
columnar aggregate of mantle-zone lymphocytes can be seen to extend into one of the channel-like spaces; another is visible at the left side of FIGURE 4 immediately above the tingible body macrophage.

Depending on the reactivity of the germinal center tissue to previous antigenic stimulation, the penetrating aggregates were numerous, deeply penetrating, and obvious, or they were shorter and less numerous. In either case, however, the leading edges of the aggregates were observed to separate into individual cells or small groups of cells that invariably followed the channel-like spaces more centrally in the germinal center. Some of these spaces anastamosed irregularly, while others appeared to end in cul-de-sacs. In the latter circumstance, macrophages were observed to occlude the channel lumen (FIG. 4). With reference to FIGURES 3 and 4, it is evident that very few small lymphocytes remain in the germinal center, and those remaining are variously pycnotic or in terminal stages of autolysis. The tingible body macrophages contained the degenerate remains of many of them.

Except for the mantle-zone small lymphocytes, already referred to, the capacious germinal center (FIG. 3) consisted of irregular masses of medium-sized lymphocytes, large lymphocytes, hemocytoblasts, macrophages, and pale, stellate, undifferentiated reticular cells, their greatest occurrence being in the order listed. Examples of these cell types are definable in FIGURE 4: To what extent the cleftlike channels seen in FIGURES 3 and 4 have been altered artifactually by the technical procedures used can only be surmised. In the present study, their constancy in all nodular tissue in various states of immunological reactivity, as well as with different levels of temperature control, gave credence to them as factual histological entities.

An overview of the mantle zone (FIG. 3) clearly indicates that a progressive diminution in the number of small lymphocytes has occurred from the region of the central artery to the antiarterial sector of the mantle zone. Although not discernible in FIGURE 3, the proportion of normal viable-appearing small lymphocytes to pycnotic degenerating ones appeared to decrease progressively as the antiarterial zone was approached. This feature, together with a reduction in number of small lymphocytes, appeared to unmask a cellular network of predominately medium-sized lymphocytes resembling those of the germinal center (FIG. 4). FIGURE 5 demonstrates the cell distribution and proportion of cell types that are present in the area of the mantle zone in the proximity of the central artery and in which little pycnosis has occurred. A large hemocytoblast, clusters of normal small lymphocytes, and scattered examples of medium-to-

FIGURE 3. A Group I $37^{\circ}$ nodule. Relatively few small lymphocytes remain in the germinal center. The ollular composition of the latter consists of medium-sized lymphocytes, hemocy toblasts, macrophages, and reticular cells. Channel-like spaces, some containing cords of compact lymphocytes, interrupt the cellular medium. The squared area contains two tingible body macrophages along with several empty channels and others containing invasive cords of small lymphocytes derived from the mantle zone (MA.Z.). The compact cords of small lymphocy tes around the central artery (A) progressively diminish towards the antiarterial sector (arrow). Opposite this sector, numerous small lymphocytes pass through the marginal zone (M.Z.). $\times 100$.

FIGURE 4. A higher-power view of the squared area in FIGURE 3. Two tingible body macrophages (T.M.P.G.) are compactly surrounded by medium-sized lymphocytes and hemocy toblasts (HC.BL.). Channel-like spaces occluded by occasional macrophages (MPG.) and others occupied by compact cords of small lymphocytes are evident. $x 315$. 

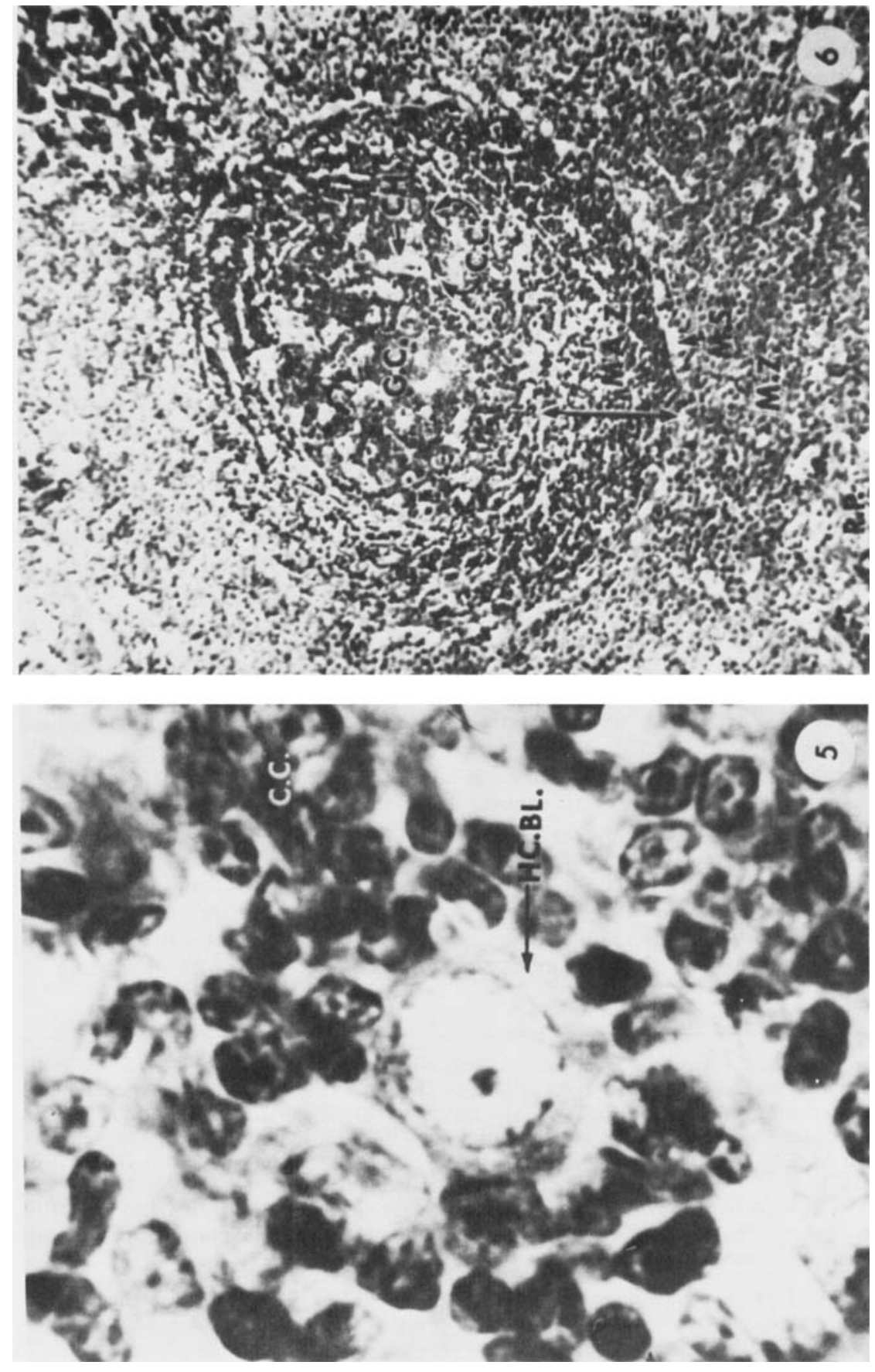
large lymphocytes can be identified. The circumscribing mass of small lymphocytes relative to the hemocytoblast (FIG. 5) was observed to penetrate for a considerable distance into the adjacent germinal center tissue in a manner identical to that of the penetrating aggregates shown in FIGURE 4.

The thin, irregular, cleft-like space peripheral to the mantle zone (FIGS. 3 and 6) represents the marginal sinus, a structure which is peculiar to the rat. This sinus is partially lined with endothelium $;^{38}$ it may be a continuous blood space or it may be discontinuous. It received numerous small nodular arterioles as well as occasional arterioles from the penicillar arteriolar complex. Moreover, the marginal sinus appears to drain into the parenchyma of the marginal zone in which blood becomes sequestered without an established provision for its return to the venous system. The marginal sinus is restricted to the bulging eccentric part of the splenic nodule and ceases at a variable point where the nodule merges with the white pulp (FIGS. 2, 3, 15).

Attention is directed to FIGURE 6, the nodule of which has been sectioned in a plane perpendicular to that of FIGURE 3. The plane of section has passed through the antiarterial sector of the mantle zone and thus shows a marginal sinus that completely circumscribes the nodule. Inclusion of the distal tip of the germinal center tissue in FIGURE 6 provides a more favorable perspective of the interrelationship of mantle zone with germinal center tissue than could be obtained from FIGURE 3 alone. It should be noted that the majority of small lymphocytes in FIGURE 6 are pycnotic and that they form a continuum through the mantle and marginal zones into the red pulp.

Group I $30^{\circ} \mathrm{C}$. Depression of splenic temperature to $30^{\circ}$ by extracorporeal means continued to show little change in shape or size of the splenic nodules and of the white pulp when compared with normal spleens or $37^{\circ}$ spleens. A comparison of FIGURE 7 with FIGURE 3 indicates, however, that prominent histological alterations occurred between $37^{\circ}$ and $30^{\circ}$. Small lymphocytes reveal marked depletion in the periarterial areas of the white pulp, and no discrete mantle zone remains. The germinal center tissue has become so sparse and diffuse that it can no longer be identified as a separate area within the nodule (FIGURE 7). With higher power than that shown in FIGURE 7 , the poorly delineated marginal sinus contained debris of small lymphocytes in some areas; in others it was compressed and empty. Although the foregoing changes in the histology of the $30^{\circ}$ spleen manifested cell depletion through cell destruction, it should be emphasized that in each zone or area of the nodule, normal viable-appearing cells remained, especially in the immediate vicinity of the central artery and in local areas of the germinal center.

The very ample marginal zone retained a normal cyto-architecture. Its cells,

FIGURE 5. High-power view of the cellular composition of a compact cord seen in FIGURE 4. Numerous small lymphocytes, including a few medium-sized forms, compactly surround a hemocy toblast (HC.BL.). $\times 690$.

FIGURE 6. A Group I $37^{\circ}$ nodule in which the plane of section is at 90 degrees variance with that of FIGURE 3. The plane of section passes through the thin antiarterial sector of the nodule. The marginal sinus (M.S.) completely surrounds the nodule in this plane of section. Only a small portion of the germinal center (G.C.) is included. Channel-like spaces (CH.), many of which contain compact cords of small lymphocytes (C.C.) derived from the mantle zone (MA.Z.), are visible. A continuum of pycnotic small lymphocytes in large numbers is obvious from the marginal sinus (M.S.) through the mantle zone (M.Z.) into the red pulp (R.P.). $\times 100$. 

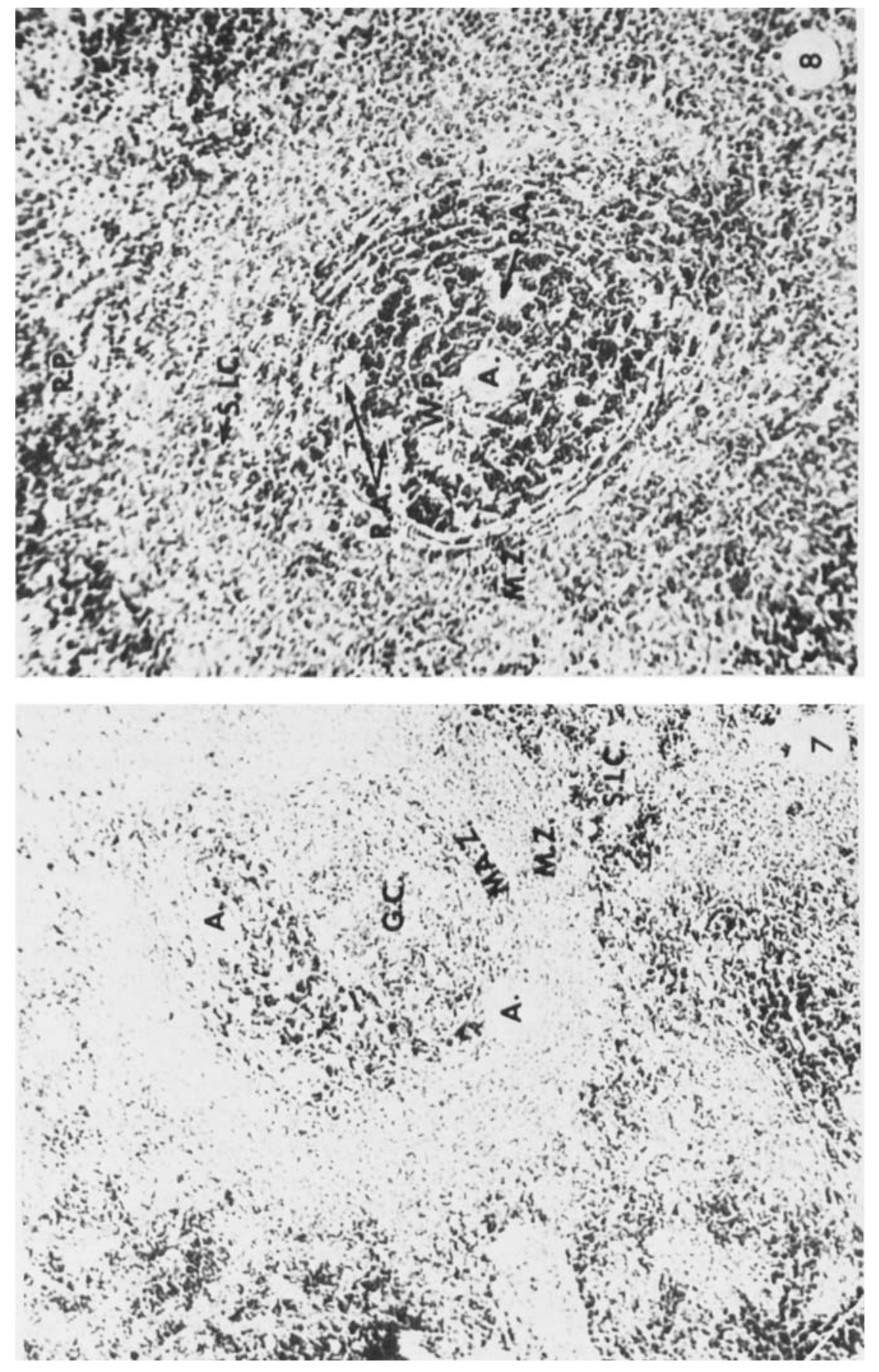
consisting of modified medium-sized lymphocytes, formed clusters and short columns. These cells revealed a progressive decrease in affinity for pyronine from the region of the marginal sinus to the junction with the red pulp. All sectors of the marginal zone included pycnotic small lymphocytes, but rarely to the extent of that found in $37^{\circ}$ spleens (FIGURE 6). It is of interest to note, however, that in the $30^{\circ}$ spleen the greatest accumulations of small lymphocytes do not lie within the confines of the nodule but occupy a region peripheral to it in the red pulp (FIGURE 7). The white pulp and surrounding marginal zone in FIGURE 8 is also from a $30^{\circ}$ spleen. The larger voids in the white pulp represent cell-depleted areas from which lymphocytes have been released or have undergone degeneration. The thinner, irregular, crack-like spaces that surround compact masses of small lymphocytes suggest shrinkage or agglutination artifacts. Several carefully controlled attempts to eliminate them met with failure. Considerable numbers of both pycnotic and normal small lymphocytes are scattered throughout the marginal zone. In selected areas (top of FIGURE 8 ), continuous bands of these cells extend from the white pulp through the marginal zone in unbroken continuity and continue into the red pulp. Occasional hemocytoblasts similar to those seen in FIGURES 4 and 5 were observed among the cell bands just described.

Spleens maintained at $30^{\circ}$ revealed marked congestion of the red pulp. Venous sinuses and intersinus areas contained closely compacted red blood cells. Many of the latter were undergoing destruction. Numerous hemosiderin-laden macrophages within both the sinuses and the intersinus areas showed a greater than normal rate of blood destruction. When the spleen was eased out of the thermal end capsule at the conclusion of exposure to $30^{\circ}$, it was observable that the organ was firm, dark red in color, and triangular in shape. This was in contrast to the paler color and the more flaccid state of the spleen when it was initially exteriorized from the body cavity and prior to its insertion into the thermal end capsule. Trabecular smooth muscle generally was in marked contraction. The features just described persisted and were accentuated in Group I spleens that were exposed to temperatures below $30^{\circ}$.

Group $I 26^{\circ} \mathrm{C}$. Artificial temperature depression of $11^{\circ}$ below body temperature produced several noteworthy histological changes in the splenic nodular complex. A comparison of a $37^{\circ}$ nodule (FIGURE 3) with a $26^{\circ}$ nodule (FIGURE 9) reveals a superficial resemblance between the two, whereas a comparison between a $30^{\circ}$ nodule (FIG. 7) and a $26^{\circ}$ nodule (FIG. 9) exhibits a marked histological difference in spite of a lesser range of temperature variation. It will be noted that in the $26^{\circ}$ nodule a continuous layer of perifollicular cells

FIGURE 7. A Group I $30^{\circ}$ nodule. The release of small lymphocytes and/or their degeneration products from the nodule has severely depleted the mantle zone (MA.Z.). The tissue of the germinal center (G.C.) is diffuse, and its borders are poorly defined. Scattered cords of small lymphocy tes remain in the periarterial regions of the nodule. Compact masses of small lumphocy tes (S.LC.) form an irregular wreath at the periphery of the marginal zone (M.Z.). The wreath is broadest opposite the antiarterial sector of the nodule. $\times 75$.

FIGURE 8. An area of white pulp from a Group I $30^{\circ}$ spleen. The white pulp (W.P.) peripheral to the central artery (A.) has the appearance of a checkerboard. Compact masses of small lymphocy tes alternating with rarefied areas (R.A.) from which lymphocy tes have been released to the marginal zone (M.Z.) and red pulp (R.P.) produce this effect. Emissions of small lymphocytes (S.LC.) from the white pulp are visible in all quadrants of the marginal zone. $\times 90$. 
has become hypertrophied (indicated by arrows in (FIGS. 9 and 10). The hypertrophied cells represent the "rind" cells of Krumbhaar, 25 which he referred to as a young type of fibroblasts, or the "reticulum cells" of Marshall. 28 Marshall included the reticulum cells in his metalophil system since these cells were blackened by silver impregnation, but he maintained an equivocal attitude regarding their functional relationship to the reticuloendothelial system. Snook, ${ }^{37}$ who found "an unusual aggregation of cells at the margin of the white pulp and in close association with the marginal sinus, "regarded these cells as marginal metalophils." He further noted that the majority of the marginal metalophils appeared to be "draped around the marginal sinus along the follicular surface of the nodule." In the present study, the hypertrophied cells, previously referred to, correspond precisely with the location of the marginal metalophils reported in Snook's studies.

Although silver impregnations were not attempted in the present study, the term "marginal metalophil" will be used to designate the cell aggregates (hypertrophied or nonhypertrophied) that lie in relation to the marginal sinus as described by Snook. ${ }^{38}$ In $37^{\circ}$ and $30^{\circ}$ spleens the marginal metalophils appeared as somewhat flattened, pale cells occasionally overlapping one another on the nodular surface of the marginal sinus. The cytoplasm appeared homogeneously opaque and stained feebly with pyronin. The nucleus was chromatin-poor to the extent that the nuclear membrane and nucleolus stood out boldly in spite of its feeble affinity for methyl green. Mitotic figures were conspicuously absent. Few cytoplasmic processes were seen in the marginal metalophils immediately adjacent to the marginal sinus; however, processes were frequently seen on the nodular surface, where they graded into and contacted stellate reticular cells more centrally located within the nodule. The cellularity of the nodular tissue in $37^{\circ}$ spleens was so intense that a full view of a single marginal metalophil was difficult, if at all possible. Examples of these flattened and often indistinct cells are seen in FIGURES 16, 18, and 22 (indicated by arrows).

The prominent hypertrophy of marginal metalophils in $26^{\circ}$ spleens, previously mentioned, not only was striking in itself but also appeared to underwrite other coincident major histological changes. Attention is directed to the presence of an irregular mantle zone in a $26^{\circ}$ spleen (FIG. 9), while in FIGURE 7, which shows a $30^{\circ}$ spleen, no distinct mantle zone remains. Reference to germinal center tissue in both these figures indicates that dissipation of the products of incomplete autolysis, as well as viable cells, is more complete in the $30^{\circ}$ spleen than in its $26^{\circ}$ counterpart. In FIGURE 10 it appears that the row of abutting hypertrophied marginal metalophils has in effect produced the restraint of a somewhat permeable dam to cellular products seeking to leave the nodule proper. Arrows in FIGURE 10 indicate sites where egress of columns of small lymphocytes is still possible. It is noteworthy that hypertrophied marginal metalophils are restricted in location to areas of the nodule that include the marginal sinus. At the termination of the marginal sinus on either side (FIG. 2) a flattened layer of modified metalophils extends around the white pulp and its vascular contents to complete the envelopment of this sector of the nodule. The modified metalophils resemble the flattened cells indicated in FIGURES 16, 18, and 22 (indicated by arrows).

Hypertrophied marginal metalophils were usually rounded in shape although a few tended to be more trapezoid in appearance. The expansive cytoplasm was highly vacuolated with vacuoles of uniform size. A pale-amber-colored material contained in the vacuoles suggested the inclusion of a lysate of hemoglobin. The 
vacuolar appearance of the cytoplasm may be seen in FIGURE 10 but is more clearly seen in a Group I $15^{\circ}$ spleen in FIGURE 14 . The hypertrophied marginal metalophils did not appear to be phagocytic. No particulate substance was observed in the vacuoles in addition to the amber-colored material. The bloated condition of the cells suggested that imbibition of materials had occurred rather than through phagocy tosis per se.

In FIGURE 10 it will be noted that the marginal sinus in its usual relationship to the hypertrophied marginal metalophils is distorted and that it communicates freely with masses of blood cells in the marginal zone. The latter observation confirms early disrupture of the marginal zone. At various points in the marginal zone it could be determined that the marginal sinus was conducting blood through the marginal zone directly to the red pulp. This flushing effect on the cellular contents of the marginal zone appeared to inhibit the buildup of masses of lymphocytes and their degeneration products at the junction of the marginal zone with the red pulp in Group I $26^{\circ}$ spleens in contrast to Group I $30^{\circ}$ spleens (FIG. 7), in which the marginal zone more nearly retained its normal structure and function.

Group $I 15^{\circ} \mathrm{C}$. Subjection of spleens to artificial temperature depressions of $15^{\circ}$ invariably led to irreversible damage. It appeared necessary, however, that this temperature exposure also be included in the study in order that the histological events leading to this point might also be ascertained. The effects of temperature exchange between the thermal end capsule and the spleen were revealed in progressive histological change from the periphery of the organ to its central core. Minimal gradations of histological change occurred in $37^{\circ}$-to- $30^{\circ}$ spleens, while more obvious stepwise gradations were displayed in $26^{\circ}$-to-15 spleens. In Group I $15^{\circ}$ spleens, however, histological gradations of nodular tissue and white pulp were virtually eliminated; the histological effects of cold being uniform throughout the spleen except for restricted foci in the vicinity of central arteries. For purposes of description, a decisive "mean point" of change was uniformly selected between extremes in change.

FIGURE 11 depicts the histological structure of the vast majority of nodules in spleens exposed to $15^{\circ}$. A loose grouping of stellate cells adjacent to the central artery indicates the remnants of the germinal center tissue. A mixture of pycnotic small lymphocytes and nuclear debris occupies the remainder of the central region of the nodule. Insert B of FIGURE 11 is shown at greater magnification in FIGURE 13. The stellate cells represent the degeneration residue of the germinal center parenchyma. They resemble medium-to-large-sized lymphocytes for the most part but also include a few degenerate forms of stellate reticular cells. Very few supportive stellate cells appear to form a background tissue in the mantle zone; the latter consisting primarily of pycnotic small lymphocy tes (FIG. 13). It must be assumed that the supportive network of modified medium-sized lymphocytes and reticular cells normally present has undergone involution. In FIGURE 11 the mantle zone appears disorganized and indefinite. Although the great majority of the small lymphocytes in the mantle zone are pycnotic, a small number of normal-appearing cells were observed to persist among them and in the minimally remaining white pulp immediately surrounding the central artery.

The marginal sinus was identifiable as a sinus only in isolated sectors of the nodule; elsewhere the mantle and marginal zones merged. A limited portion of the marginal sinus is shown in FIGURE 12. The relationship of marginal metalophils to the sinus was irregular (FIG. 12, insert A). In other sectors of the nodule, marginal metalophils were absent or failed to form a contiguous layer. In 


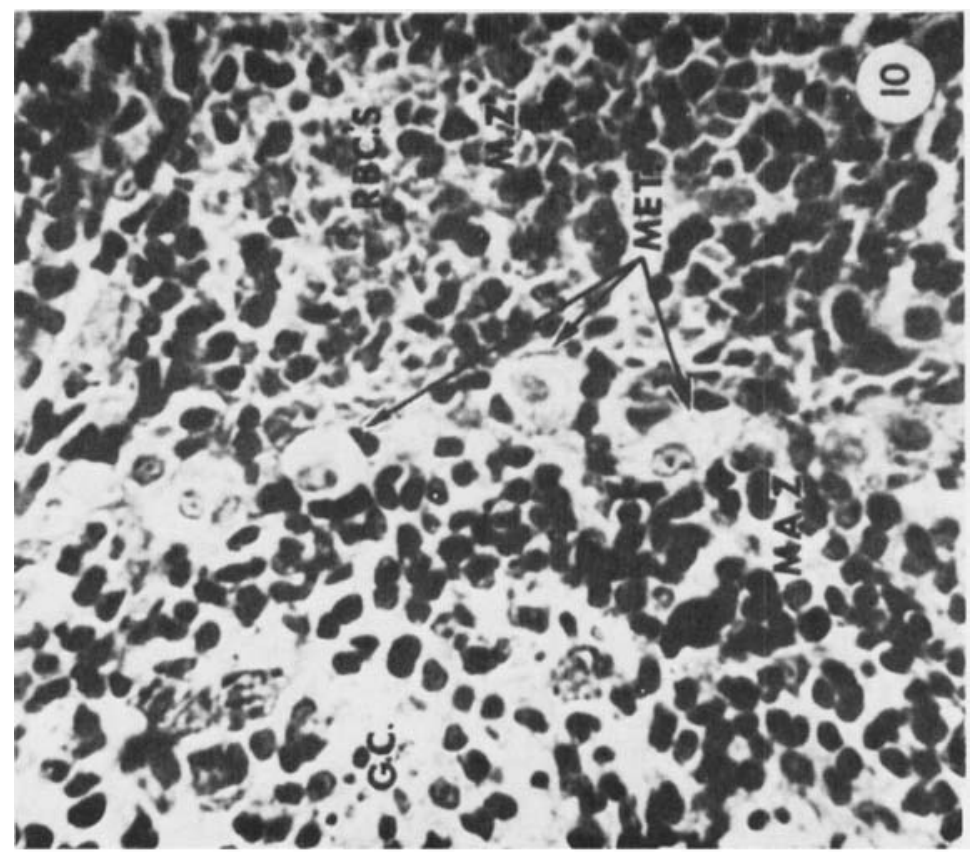

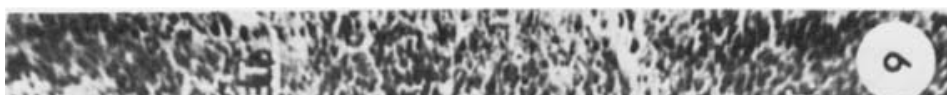

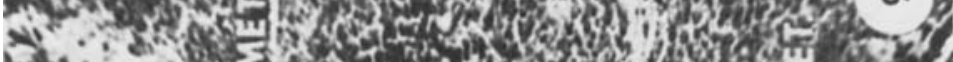

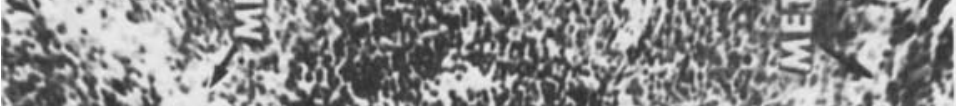
c. ${ }^{2}$.

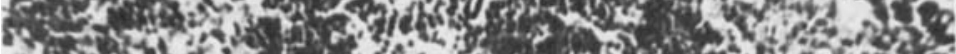

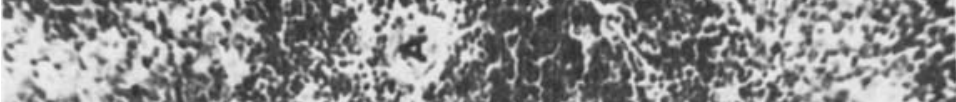

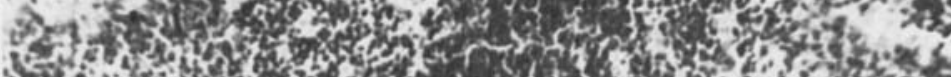

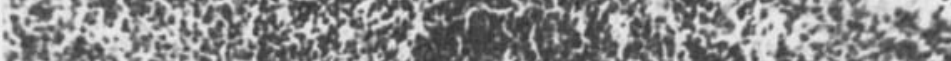

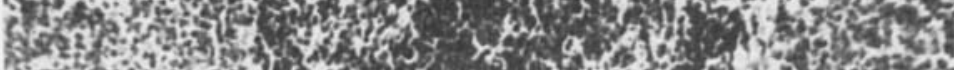
(2)

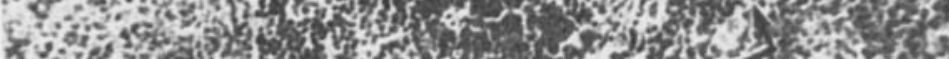

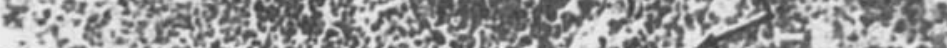
$\because$ tor

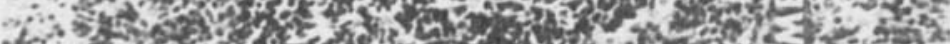

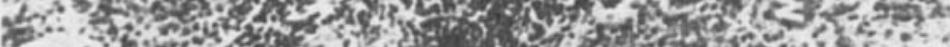

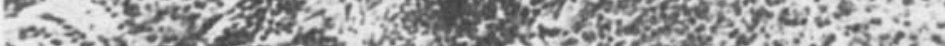

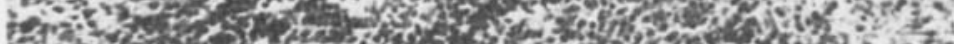
Far

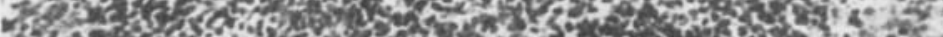

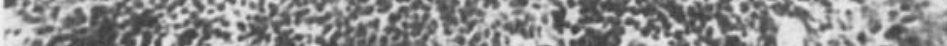

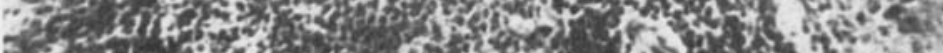

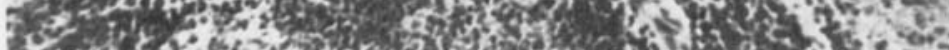


this latter instance, small pycnotic lymphocytes appeared to disperse from the mantle zone into the marginal zone without restraint (diagonally left of asterisk, FIG. 11). As a result, considerable numbers of pycnotic lymphocytes together with red corpuscles occupied the depleted marginal zone parenchyma to form a continuum from the marginal sinus to the red pulp. It is interesting to note that only occasional instances of marginal metalophil hypertrophy occurred in Group I $15^{\circ}$ spleens. That it did occur, however, is illustrated in FIGURE 14, a view of a nodule located well within the central core of the spleen. Small, uniform vacuoles pervade the cytoplasm and its processes. None of the vacuoles were observed to contain particulate matter.

The volume proportions of red pulp to white pulp in Group I spleens remained normal and constant from $37^{\circ}$ to $30^{\circ}$ although congestion of the red pulp was evident. An abnormal increase in the number of hemosiderin-laden macrophages was more pronounced in $30^{\circ}$ spleens than in other Group I spleens. Variation in volume proportion of red and white pulp was accentuated progressively from Group I $26^{\circ}$ spleens to those at $15^{\circ}$. White pulp was progressively reduced between these temperature levels in ratio to red pulp. Venous sinuses contained a reduced number of blood cells at $15^{\circ} \mathrm{C}$ in comparison to those at $26^{\circ} \mathrm{C}$. An obvious decrement in hemosiderin-containing macrophages also was observed to occur between these two temperature levels.

\section{Group II. (Three Hours of Regulated Temperature; Spleen Reperitonealized for 72 Hours prior to Splenectomy). Groups I and II compared}

Group II $37^{\circ} \mathrm{C}$. The morphology of the nodular tissue, zone for zone, as well as its histological characteristics (FIGS. 15 and 16) closely resembled that of normal nodules randomly selected from animals that had not been subjected to artifical temperature control. In Group II $37^{\circ}$ spleens a broad, highly cellular mantle zone was evident (FIG. 15). Blunt centripetal projections of mantle zone lymphocytes into the germinal center both reduced the size of the germinal center and altered its shape. Comparison of a Group $137^{\circ}$ nodule (FIG. 3) with a Group II $37^{\circ}$ nodule (FIG. 15) indicates that the mantle zone of the former is more diminutive, that its germinal center is larger, and that definition of its margin is more distinct. Both nodules (FIGS. 3 and 15) were cut near their

FIGURE 9. A Group I $26^{\circ}$ nodule. Arrows point to hypertrophied marginal metalophils (MET.) in a nodule that has been centered in the Figure and in two others partially included. Hypertrophy of these large, pale-staining cells is usually most pronounced in the antiarterial sector of the nodule. They were never observed to extend beyond the limits of the marginal sinus. An irregular but definable mantle zone persists. Compare with FIGURE 7. $\times 75$.

FIGURE 10. A higher-power view of hypertrophied marginal metalophils (MET.) in the antiarterial sector of a Group I $26^{\circ}$ nodule. The germinal center (G.C.) and the mantle zone (MA.Z.) lie to the left of the vertical row of marginal metalophils; to the right is the marginal zone (M.Z.). Small uniform cytoplasmic vacuoles fill the cytoplasm of hypertrophied metalophils (see also FIGURE 14). Hypertrophy of marginal metalophils impedes the release of small lymphocytes from the germinal center and mantle layer. Note the rowlike columns of small lymphocy tes passing between adjacent metalophils. Note also that the indefinite marginal sinus is openly conducting blood (RBC'S) through a partially destroyed marginal zone (M.Z.). $\times 315$. 


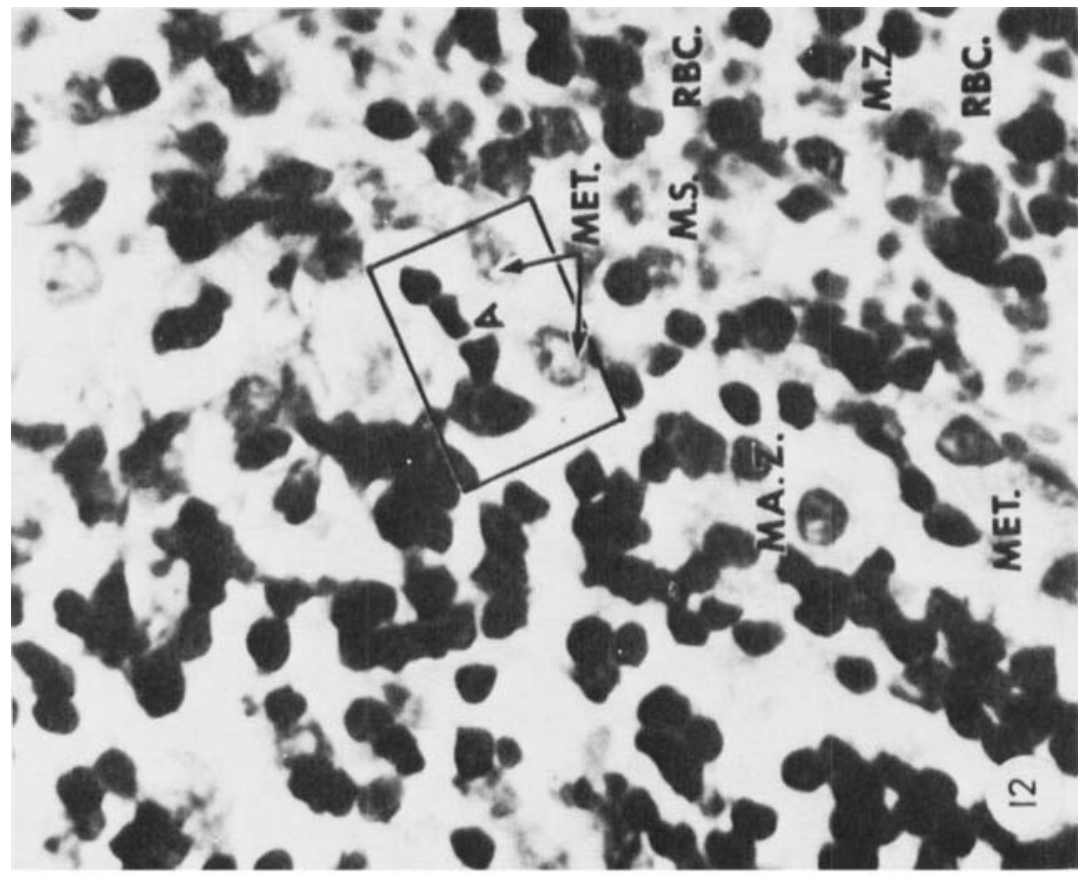

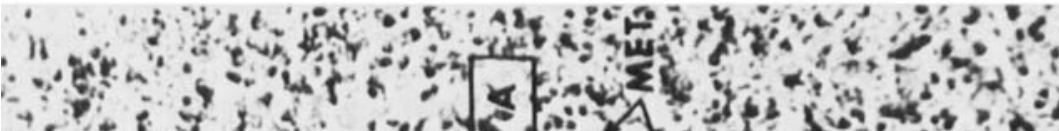

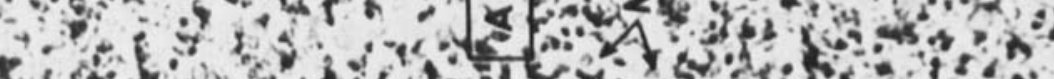

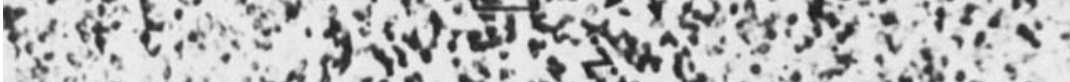

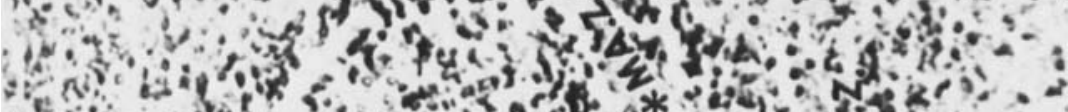
4. if $x^{2}$, ?

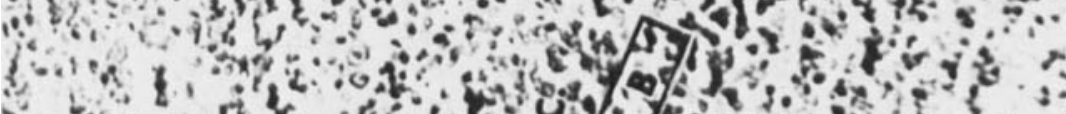

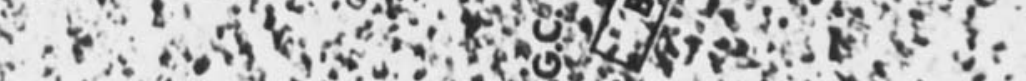

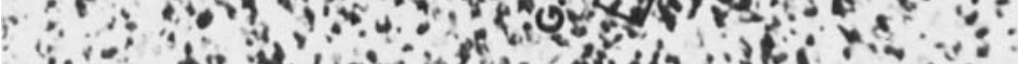

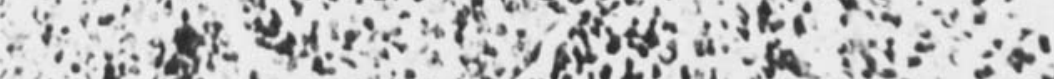

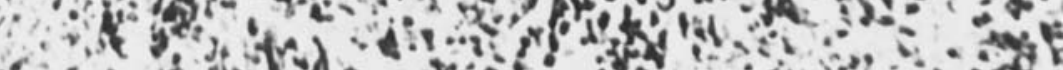
of $x_{12}$. 2

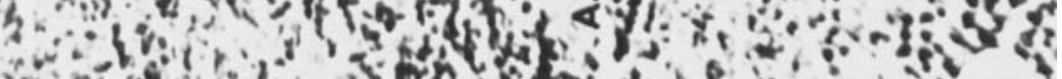

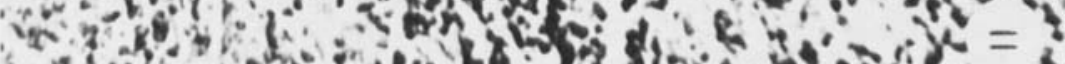
anes? 
center in a nearly identical plane. The obvious thin point in the mantle zone (indicated by an arrow, FIG. 3) is more difficult to detect in the more cellular mantle zone (arrow, FIG. 15). From the foregoing, it appears that the mantle zone lymphocytes were reconstituted during the space of 72 hours in $37^{\circ}$ Group II spleens.

It should be recalled that relatively marked depletion of mantle zone lymphocytes occurred in Group I $37^{\circ}$ spleens by means of progressive fragmentation and autolysis of the cells as well as by phagocytosis. The marginal zone and adjoining red pulp contained considerable debris of these degenerating cells (FIGS. 3, 4 and 6). It will also be recalled that, in the periarterial white pulp in all Group I spleens, normal small lymphocytes were always present, and that these cells extended into the mantle zone in varying numbers, depending upon the level of temperature exposure utilized. The infrequent occurrence of mitoses in the small lymphocytes could not in itself justify the obvious increase in their number that occurred during mantle zone reconstitution. Compact cords of small lymphocytes lateral to the central arteries (FIG. 15), when followed serially, were observed to have their origins in anastomosing cords of identical cells deep within the white pulp. The cords were commonly observed to lie parallel to thin-walled vessels in whose lumen small lymphocytes were present. Occasional signs of lymphocyte diapedesis from these vessels was evident. This observation was in contrast to the total absence of lymphocytes or other formed elements of the blood in the lumina of the central arteries. The muscular tunics of the latter were markedly contracted, as evidenced by a much-folded luminal configuration. White pulp cords were slender and separated by a slight hiatus from adjacent cords and vessels parallel to them. Nearer the junction of the white pulp with the mantle zone, the cords first became broader and the spaces separating them less obvious, until a nearly uniform cellular medium existed in the mantle zone proper. When less than a densely packed, full compliment of small lymphocytes occupied the mantle zone (FIGS. 15 and 16), occasional irregular crowfoot crevices separated short chains, groups, and aggregates of small lymphocytes. This circumstance appeared to be related to a plasmapressure-cell-volume imbalance in the increasing cell mass within the fibrocellular framework of the mantle zone as well as to linear agglutination of some of the small lymphocytes. These same observations applied to hemocy toblasts included among the aggregates of small lymphocytes.

The mantle zone of Group II $37^{\circ}$ spleens revealed only scattered examples of pycnosis (FIG. 16). Moreover, the histology of the marginal zone parenchyma exhibited little deviation from normal splenic nodules. It contained far fewer pycnotic small lymphocytes in comparison to Group I spleens (FIGS. 3 and 6)

FIGURE 11. A Group I $15^{\circ}$ nodule that, through cell depletion reveals only suggestions of its former architecture. A small group of larger stellate cells adjacent to the central artery (A.) indicates the remains of the germinal center (G.C.). A poorly defined mantle zone (MA.Z.) projects into the marginal zone (M.Z.) between the asterisk and the squared area (B.). Marginal metalophils (MET.) are scattered, pale-staining, and degenerating. The marginal zone (M.Z.) cannot be distinguished from the surrounding red pulp. $\times 110$.

FIGURE 12. A higher-power view of the squared area (A.) from FIGURE 11. Two pale-staining marginal metalophils (MET.) are indicated by arrows. Immediately above, a short column of small lymphocytes extends from the mantle zone (MA.Z.) through the indefinite marginal sinus (M.S.). The marginal sinus empties its content of blood (RBC'S) diffusely into the disorganized and cellular-depleted marginal zone (M.Z.). X 430 . 


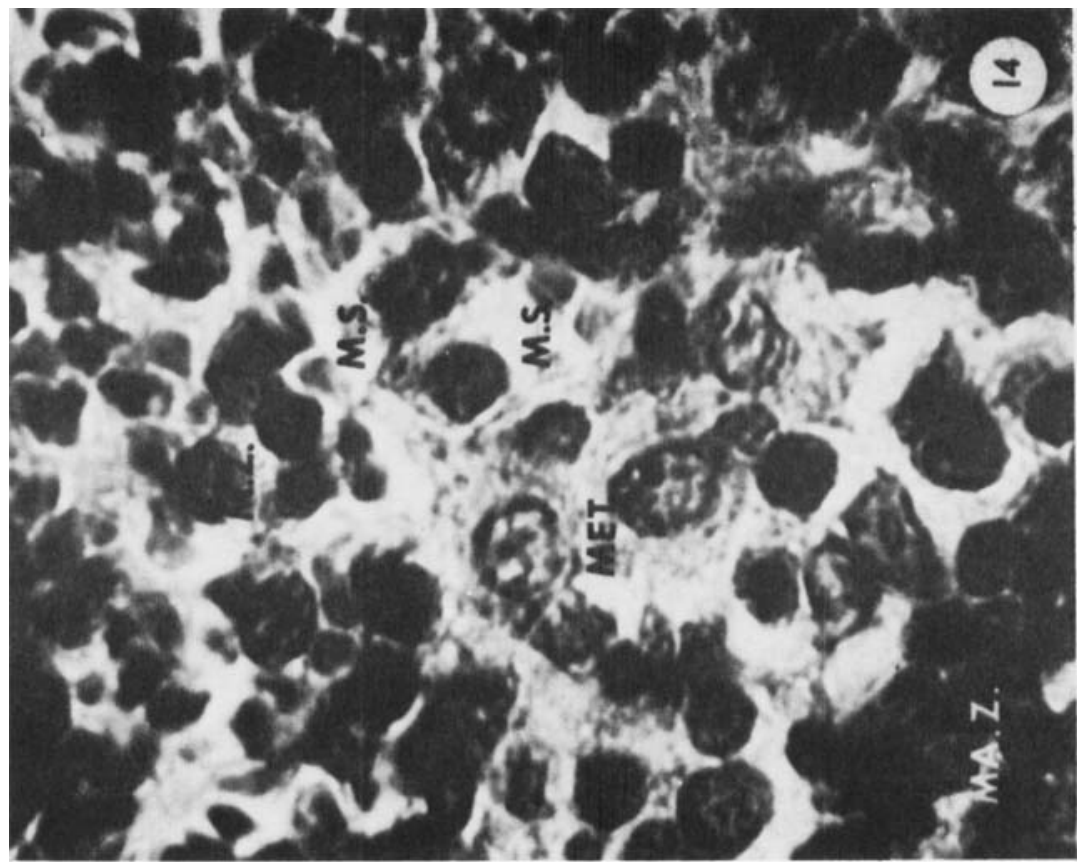

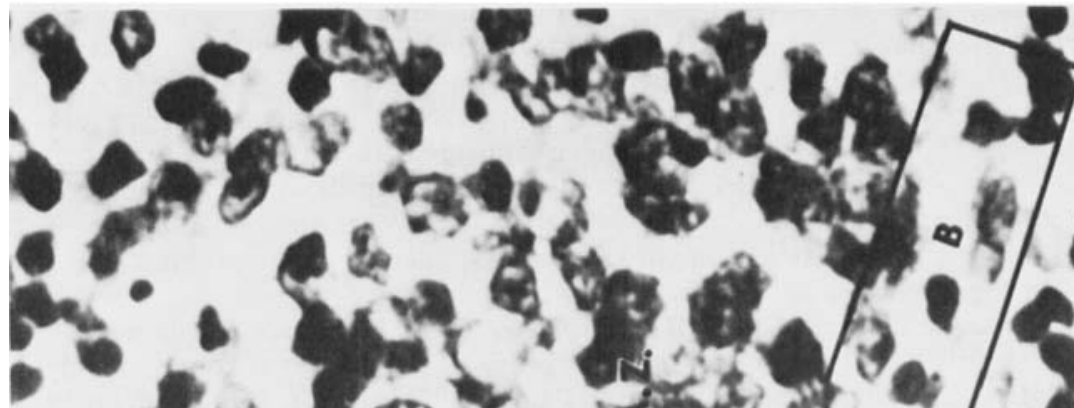

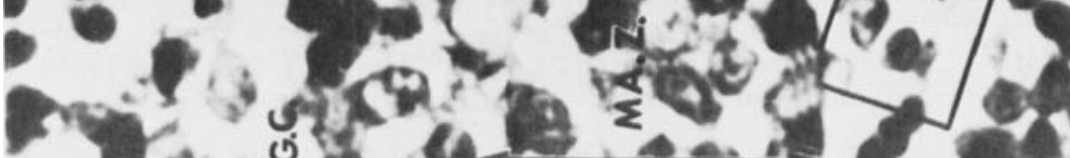

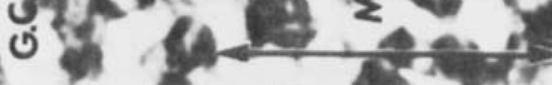

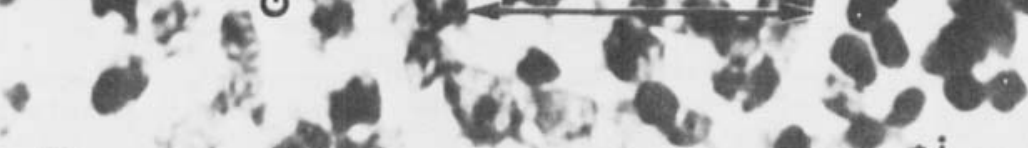

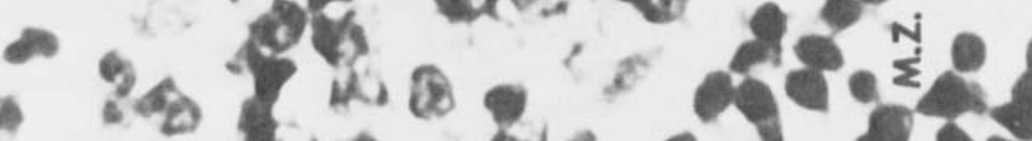

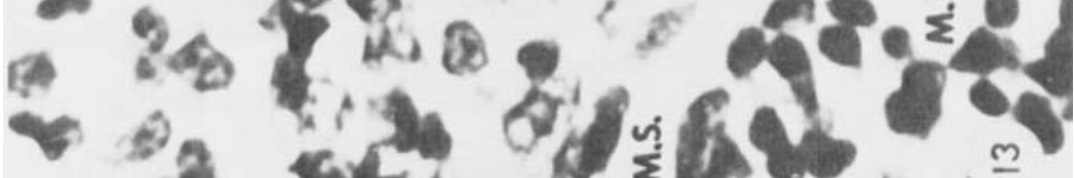
- . 
for the same temperature. Attention is called to the flattened (modified) layer of metalophils located beneath the endothelium of the marginal sinus and beyond the termination of the latter. Modified metalophils in the nonsinus sector of the nodule were not observed to undergo hypertrophy (FIG. 9) in response to temperature regulation or to restrain as effectively the egress of cells from the white pulp (arrows in upper part of FIGS. 2, 8, and 21), as was true for marginal metalophils related to the marginal sinus.

Group II $30^{\circ} \mathrm{C}$. The morphological zones and regions typical of normal nodules were more easily identifiable in Group II $30^{\circ}$ spleens (FIG. 17) than in Group I $30^{\circ}$ nodules (FIG. 7). The mantle zone in FIGURE 17 is partially reconstituted near its junction with the white pulp. Some pycnotic lymphocytes remain scattered throughout the germinal center, and some are contained in macrophages. In the absence of new incursions of lymphocytes into the germinal centers of the reperitonealized organ, rampant cell destruction appears to have terminated. Reference to FIGURE 18 (squared area of FIG. 17) will reveal the presence of normal-appearing small lymphocytes and hemocytoblasts. Very few pycnotic lymphocytes remain in the nodule. Marginal metalophils show no evidence of hypertrophy.

Group II $26^{\circ} \mathrm{C}$. With certain exceptions, Group II nodules closely resembled the histological changes described for those of Group I at the same temperature. In the former group, vigorous reconstitution of the mantle zone and white pulp resulted in irregular, radially organized excrescences of mantle zone tissue (FIG. 19), and a serpentine mantle zone-marginal sinus junction. The same reconstitution, through centripetal extensions of the mantle zone tissue, has nearly obliterated the germinal center. Intranodular plasma pressure appears to be ineffectual in producing rounded contours to the nodule in the phase of nodular reconstitution just described. Arrows in FIGURE 19 indicate a few tingible body macrophages in the poorly defined but highly cellular germinal center.

Marginal metalophils, although feebly stained, were observed to be hypertrophied though less vacuolated (FIGS. 19 and 20) than those in Group I $26^{\circ}$ (FIGS. 9 and 10). Marginal metalophils of Group II spleens appeared to form a more effective dam to the peripheral egress of cells. In FIGURES 19 and 20 only one cord of cells has penetrated the marginal metalophil barrier, while in FIGURES 9 and 10 they are commonplace.

Group II spleens in general contained little blood in the marginal sinus (arrows, FIG. 20, and MS, FIG. 22), nor was blood found uniformly distributed within the marginal zone parenchyma and adjacent red pulp. This is in marked

FIGURE 13. A higher-power view of the squared area (B) from FIGURE 11. The paucity of cells in the germinal center (G.C.) and in the mantle zone (MA.Z.) is evident. Unoccluded apertures leading from the mantle zone into the marginal sinus (M.S.) represent points of release of free cells into the sinus. Marginal metalophils are conspicuously absent, except for the two examples contained in the squared area (B). The remnants of the marginal zone parenchyma (M.Z.) can be better visualized in FIGURE 13, since this sector of the nodule contains no circulation of blood corpuscles. $X 430$.

FIGURE 14. A high-power view of an area of a Group I $15^{\circ}$ nodule located doep in the central core of the spleen. An accumulation of marginal metalophils (MET.) in a better state of preservation still reveals a uniformly vacuolated cytoplasm. A portion of the mantle zone (MA.Z.) in the lower left of the figure and a blood-congested marginal zone (M.Z.) in the upper part bear certain similarities to the Group I $26^{\circ}$ nodule in FIGURE 10. Hypertrophy of the marginal metalophils in FIGURE 14 is less pronounced. $\times 675$. 

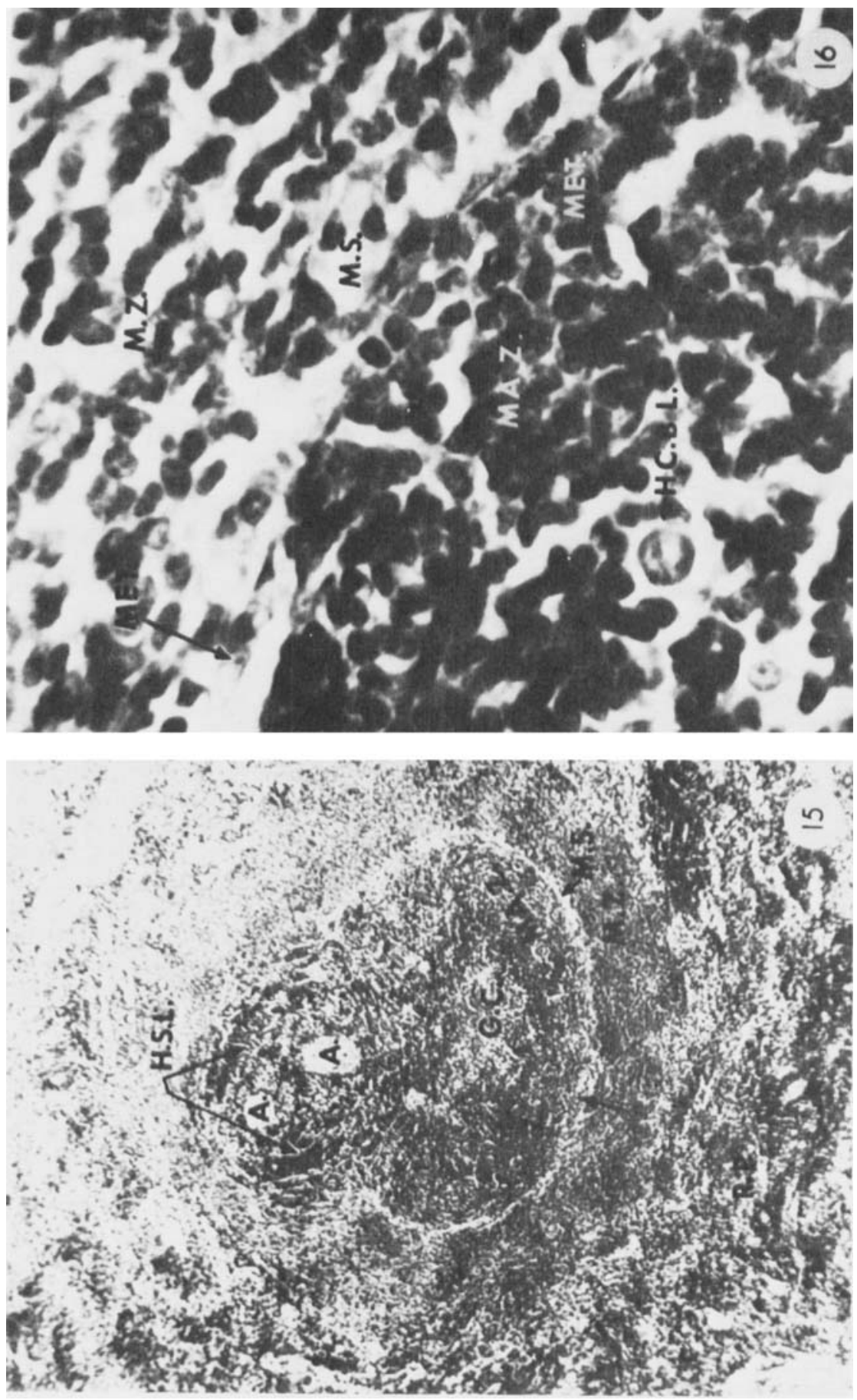
contrast to Group I spleens (FIG. 10). Peripheral dissemination of pycnotic lymphocytes and nuclear debris, so abundantly seen in Group $126^{\circ}$ spleens (FIG. 10), is also in sharp contrast to the minimal passage of more normal-appearing though hyperchromatic lymphocytes in Group II spleens (FIG. 20). The decrease in the peripheral blood content of the nodules was observed to correlate with a similar reduction in the volume of the red pulp. The decrease in peripheral blood content to which reference has been made centers on the near cessation of circulating blood corpuscles. The apparent hydration of the reconstituting mantle zone suggested, however, that a normal compliment of plasma was present. Many hemosiderin-laden macrophages still remained in the red pulp cords, indicative of earlier RBC destruction. A majority of the venous sinuses were either empty or collapsed, while the remainder contained a normal complement of blood. An obviously reduced blood content of trabecular veins also emphasized a reduction in sinusoidal circulation.

It appeared judicious to include two additional temperature decrements between $26^{\circ}$ and $15^{\circ}$ for Group II spleens in order that histological changes between these temperatures could be followed more critically. Accordingly, two spleens at $20^{\circ}$ and two at $18^{\circ}$ were studied. At $20^{\circ}$, spleens exhibited a less exuberant reconstitution of the mantle zone and white pulp (FIG. 21) than was observed in $26^{\circ}$ Group II spleens (FIG. 19). In the former, except for the mantle zone proximal to the white pulp, the majority of small lymphocytes were markedly pycnotic. Many of the cells of the germinal center were also pycnotic or failed to stain with typical specificity. Fragmented nuclear debris was only occasionally encountered throughout the nodule. Marginal metalophils variously presented shrunken or swollen nuclei and damaged cytoplasm. In some instances, the latter appeared as an empty space or as a densely opaque mass. Columns of small pycnotic lymphocytes (circumscribed in FIGS. 21 and 22) meandered through occasional apertures between damage-altered metalophils into and through the marginal zone to reach the red pulp. A greater number of small lymphocytes were observed to extend into the red pulp from the white pulp proper (arrows, top of FIGS. 2 and 21). Little peripheral blood was observed in the marginal sinus or in the marginal zone.

Although some variability existed in the histological response of Group II spleens to $18^{\circ} \mathrm{C}$, the general pattern of response was consistent. If the nodule is visualized as a sphere, each had a dark arterial pole and a considerably lighter antiarterial pole (FIG. 23). The darker pole consisted of cords and masses of

FIGURE 15. A Group II $37^{\circ}$ nodule. A highly cellular mantle zone (MA.Z.) projects centripetally into the germinal center (G.C.). Very compact cordlike masses of hyperchromatic small lymphocytes (H.S.L.) partially circumscribe the central arteries (A). Lymphocytes from this site appear to progressively augment the increasing cellularity of the mantle zone. The anti-arterial thin sector is more difficult to define (single arrow) than in a Group 1 $37^{\circ}$ nodule (see FIGURE 3). The marginal sinus (M.S.) of the Group II nodule appears consistently broader than in its Group I counterpart. $\times 75$.

FIGURE 16. Higher-power view of a Group II $37^{\circ}$ nodule near the proximal termination of the marginal sinus (M.S.). The normal-appearing cellularity and organization of the mantle and marginal zones (MA.Z. and M.Z.) are apparent. Marginal metalophils (MET.) show no evidence of hypertrophy or the formation of cytoplasmic vacuoles. A hemocytoblast (HC.BL.) is visible at the germinal center-mantle zone junction. Very few red blood corpuscles are present in the marginal sinus or in the interstices of the marginal zone. $\times 525$. 


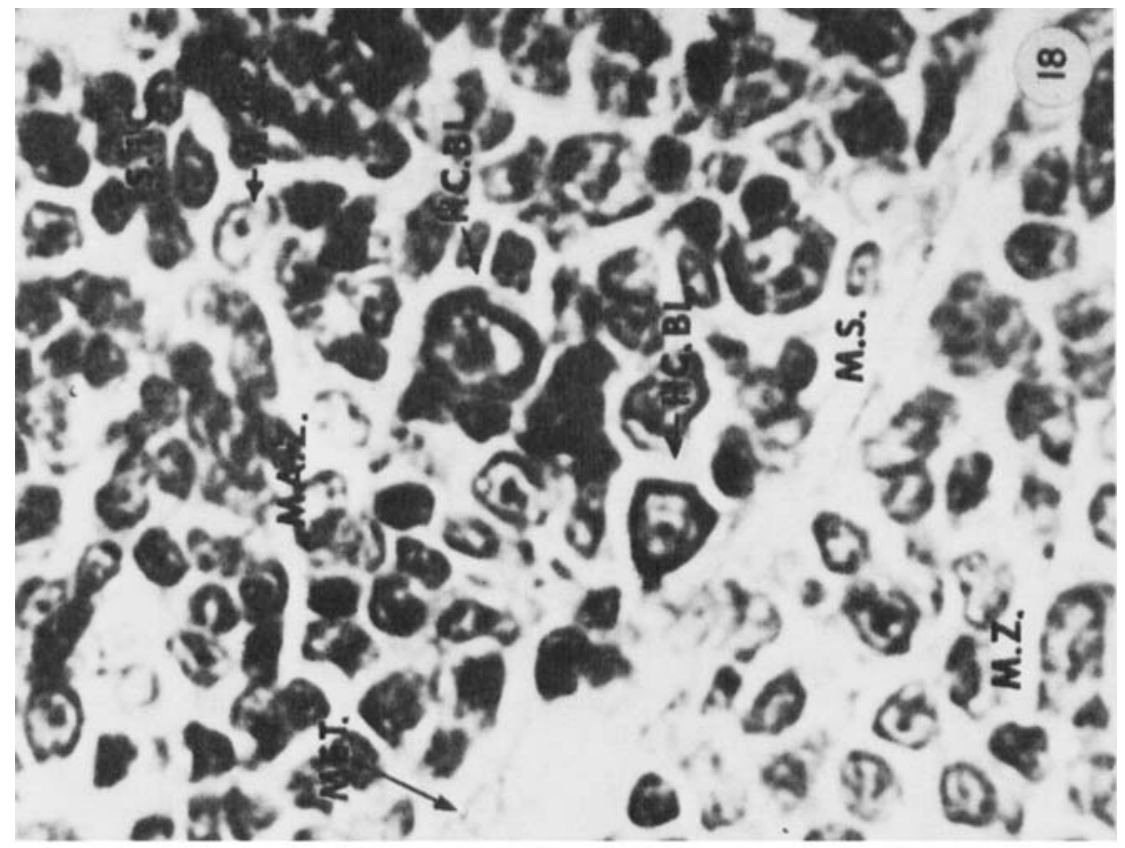

सु is 25 .

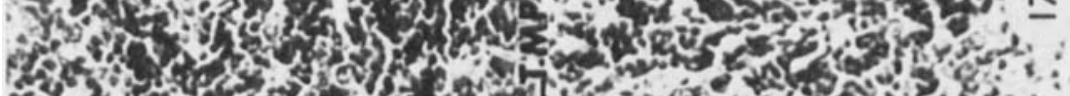

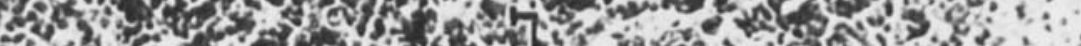
30,12 . :4. 4 .

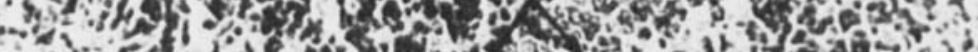

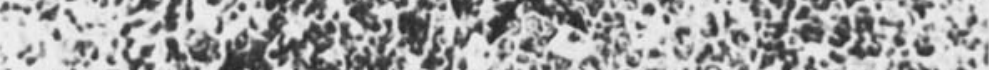

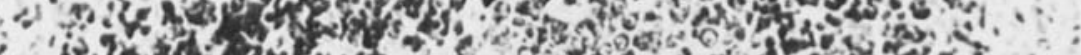

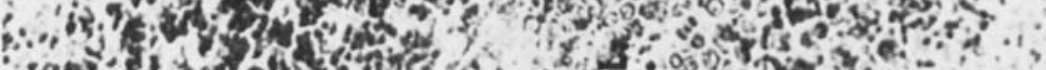

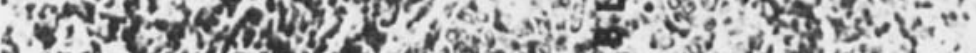

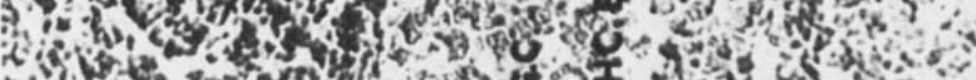

3.

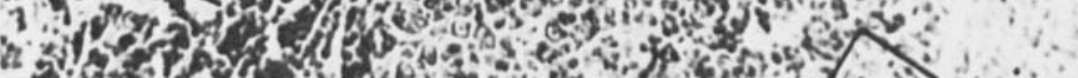

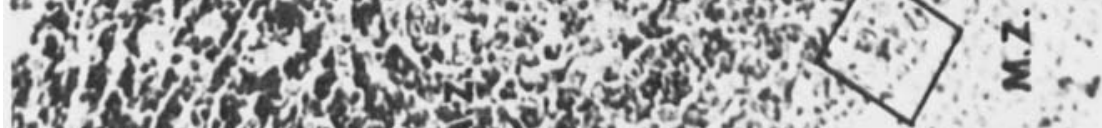

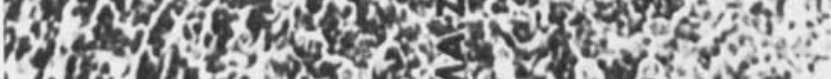

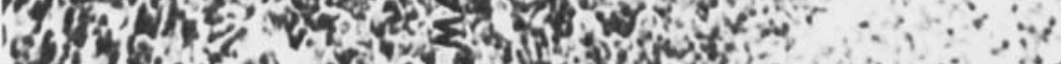

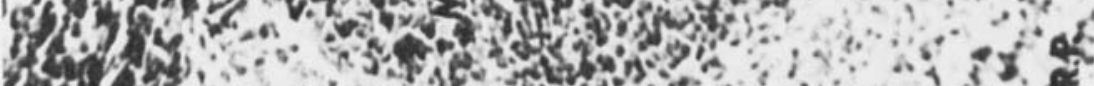

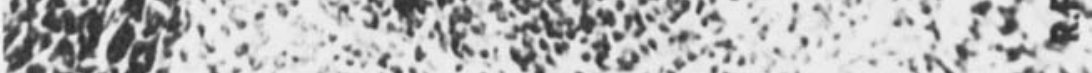
6) 
hyperchromatic small lymphocytes originating in the periarterial white pulp. The cellular masses projected in a looser form into mantle zone and germinal center areas. As a rule, the projections were not symmetrical but favored one side more than the other. The lighter pole consisted of a lesser number of hyperchromatic small lymphocytes, many of which continued diffusely through the marginal zone into the red pulp. In FIGURE 23 the marginal sinus is very broad and poorly demarcated on its marginal zone surface. The latter observation may have its basis in the partial reconstitution of the marginal zone parenchyma, or a greater than normal plasma content of the marginal sinus, or both of the foregoing.

A majority of the marginal metalophils were involuting, while others had completely disappeared. Those which remained were widely separated from one another. Cloudy, textureless nuclei surrounded by a void characterized most of them. A few, resembling those in FIGURE 12, were observed near the termination of the marginal sinus. The dam effect of these cells was no longer operative, since hyperchromatic lymphocytes, singly and in groups, appeared to be flushed from the nodule with no apparent impediment. The unbroken continuity of passage of the hyperchromatic small lymphocytes from the periarterial white pulp through the nodule and into the red pulp (FIGS. 23 and 24) clearly underscores the origin of these cells. An incomplete series of samples at $18^{\circ}$ in which the spleen was reperitonealized for 7,14 , and 21 days each revealed the same passage of hyperchromatic lymphocytes to the red pulp as has been described above for three days.

Group II $14^{\circ} \mathrm{C}$. Spleens of this group compare to Group I $15^{\circ}$. The observations made for Group II $18^{\circ}$ spleens form a substantial preface for many of the histological manifestations that occurred in Group II $14^{\circ}$ spleens. An area of white pulp and its surrounding marginal zone is shown in FIGURE 25. The white, irregular patches within the pulp are rarefied areas that usually contained a variable number of free lymphocytes. Each patch represents the site of formerly compact masses of small lymphocytes comparable to the cordlike masses seen elsewhere throughout the white pulp. With higher-power inspection, each of the patches or rarefied areas was observed to be partially occupied by stellate reticular cells, thin-walled vessels and a variable number of small, hyperchromatic lymphocytes. On the basis of the number of small lymphocytes present, many intergrades were definable among the rarefied patches. Some contained but few lymphocytes, while in others their numbers were sufficient to produce a meager cordlike cellularity.

The compact cordlike masses (FIGS. 25 and 26) consisted of hyperchromatic small lymphocytes together with smaller numbers of medium-sized forms and

FIGURE 17. A Group II $30^{\circ}$ nodule. Reconstitution of the mantle zone (MA.Z.) is progressing from the periarterial white pulp (A). The squared area represents the approximate position of the antiarterial sector. The germinal center tissue consists overwhelmingly of developing hemocytoblasts (HC.BL.). A few examples of tingible body macrophages are also present (T.MPG.). Very little cellular debris remains. Compare this figure with FIGURE 7, of a Group I $30^{\circ}$ nodule. $\times 110$.

FIGURE 18. A high-power view of the squared area in FIGURE 17. Hemocytoblasts (HC.BL.), medium-sized lymphocytes (M.LC.), and small lymphocytes (S.LC.) have approached the marginal sinus (M.S.) preparatory to entering the marginal zone. The marginal zone parenchyma (M.Z.) contains a few small lymphocytes but no hemocytoblasts. Marginal metalophils (MET.) are in a flattened, nonhypertrophied condition. $\times 545$. 

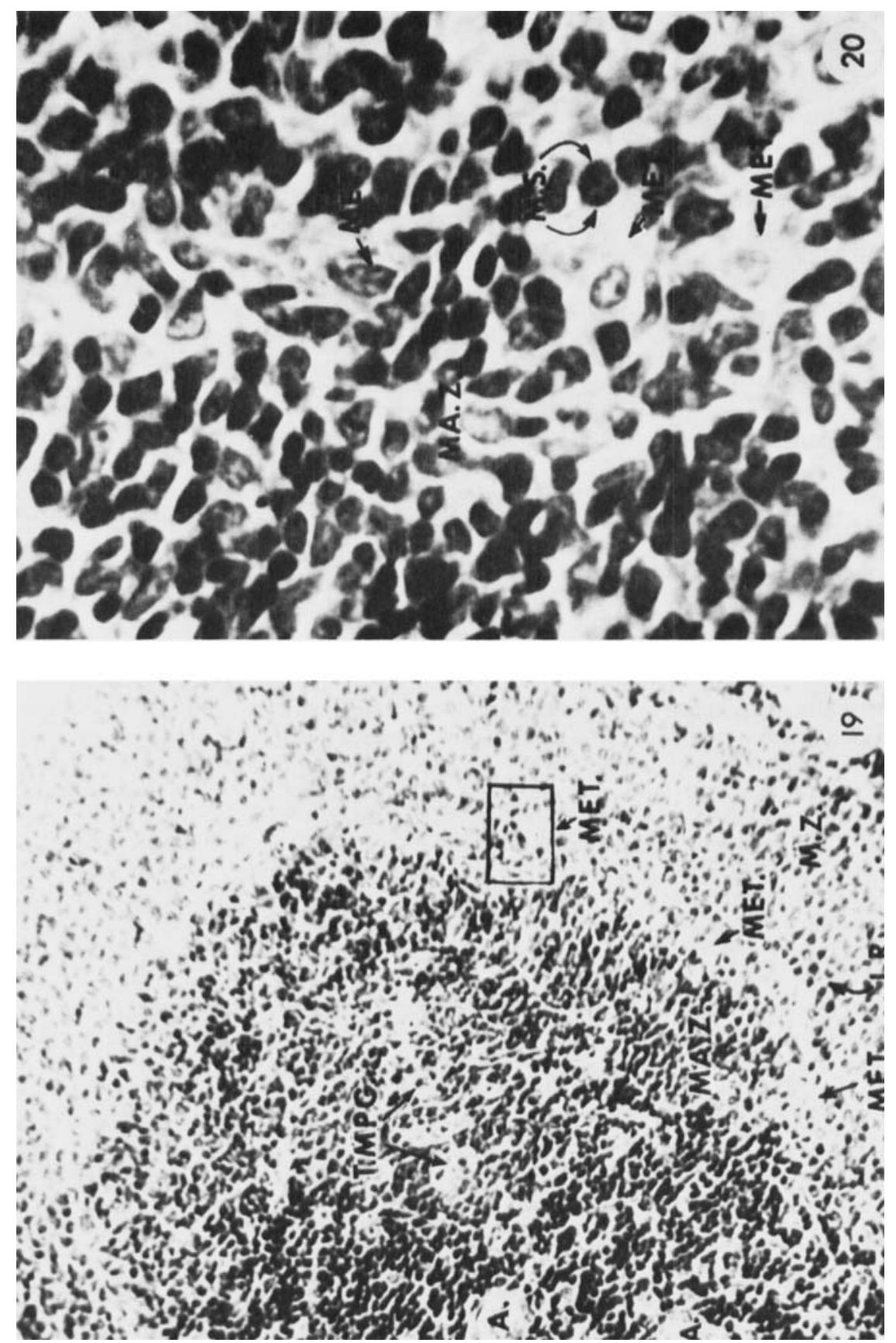
some hemocytoblast. Many of the small lymphocytes may be seen in transit from the periphery of the white pulp as they extend through the marginal zone into the red pulp (FIG. 25). Hemocytoblasts were frequently seen in company with migrating small lymphocytes. The passage of lymphocytes from the nonnodular white pulp to the red pulp was very pronounced in Group II $14^{\circ}$ spleens. It should be emphasized, however, that this feature was common in varying degree to all spleens of Groups I and II. The nodule in the upper left of FIGURE 26 is also from a Group II $14^{\circ}$ spleen. The dark pole can barely be distinguished from the former light pole. A continuing mass movement of small lymphocytes from the dark (periarterial) pole to the light (antiarterial) pole has nearly equalized the two poles (compare FIGURES 23 and 26). In the absence of a restraining layer of marginal metalophils together with possible alterations in a plasma pressure relief membrane, myriads of small lymphocytes may be seen to infiltrate the marginal zone and contiguous red pulp. Germinal centers were rarely definable in Group II $14^{\circ}$ spleens.

No essential difference could be detected in the volume proportions of red and white pulp in Group II spleens with temperature exposures of $26^{\circ}$ to $14^{\circ}$. Within this temperature range all spleens revealed a compressed red pulp in which the venous sinuses were frequently collapsed and in which blood conduction appeared acutely reduced. Blood cells were numerous in the lumina of some sinuses, while others contained none. Hemosiderin-laden macrophages in the red pulp were few in number and inconspicuous. Trabecular veins, however, always contained some blood cells of bone marrow origin, which suggested that a minimal splenic circulation remained in effect.

\section{DISCUSSION}

In the normal unoperated normothermic rat it is apparent that a complex of physical factors is at work on the spleen that influences the course of its normal functions. Among these factors are arterial blood pressure, intermittent or phasic drainage of the sinusoidal red pulp, rhythmic respiratory massage, variations in pressure of the gastric contact, and variations in intra-abdominal pressure resulting from exercise, defecation, and urination. Added to these as selective modifiers of the foregoing are elastic tissue and capsular, trabecular and arterial smooth muscle. Interplay among these factors and their effect on the cells of the

FIGURE 19. A Group II $26^{\circ}$ nodule reveals vigorous mantle zone (MA.Z.) reconstitution. Small, hyperchromatic lymphocytes surrounding the central arteries (A) have nearly obliterated the germinal center. Tingible body macrophages mark the general location of the former germinal center. Excrescences of mantle zone tissue have produced an uneven or serpentine margin. Metalophils are moderately hypertrophied (MET.). Refer to FIGURE 20. Only small amounts of nuclear debris remain in the nodule. Linear regeneration (L.R.) of the marginal zone (M.Z.) is apparent near the marginal sinus. Compare this figure with FIGURE 9, of a Group I $26^{\circ}$ nodule. $\times 215$.

FIGURE 20. A higher-power view of the squared area in FIGURE 19. Note the hypertrophied, though less vacuolated, marginal metalophils (MET.) compared with those in FIGURE 10 of a Group I $26^{\circ}$ nodule. A cord of small lymphocytes derived from the mantle zone (MA.Z.) may be observed to pass through the marginal sinus (M.S.) between adjacent metalophils into the marginal zone. The cord arises from one of the excrescences described in FIGURE 19. Very few red blood corpuscles are visible in either the marginal sinus or marginal zone (left in the figure). $\times 430$. 


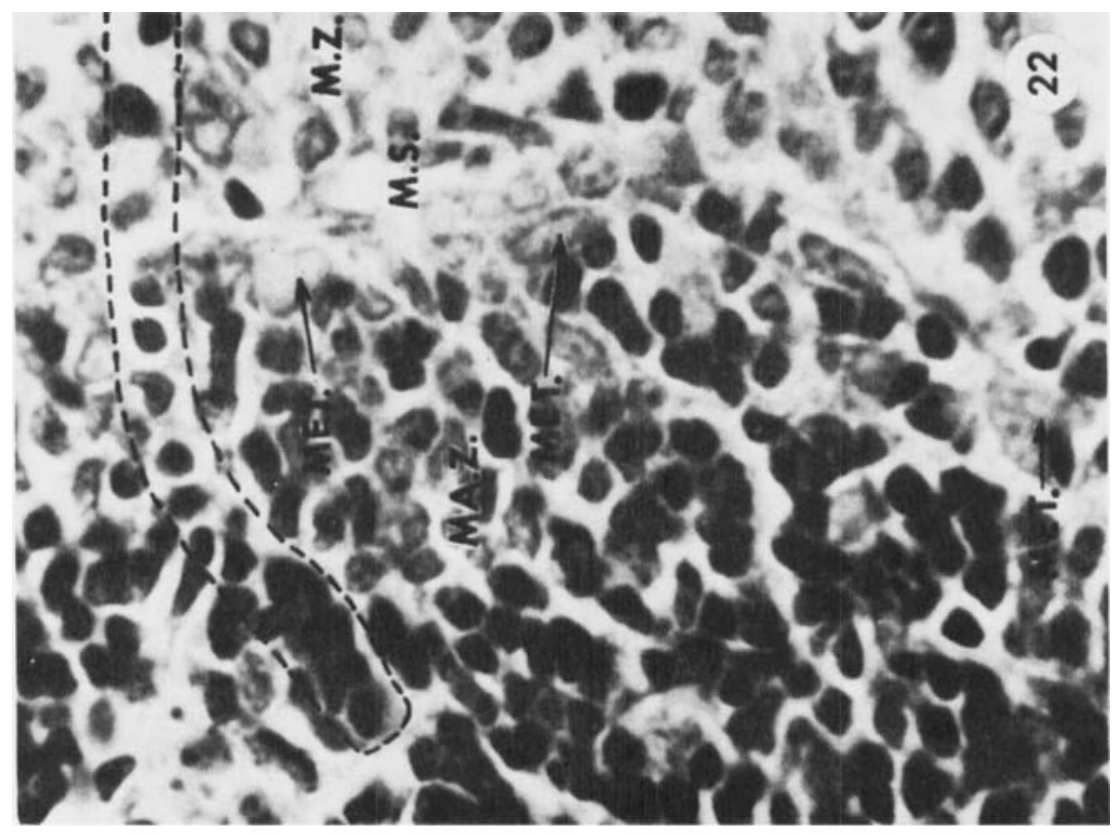

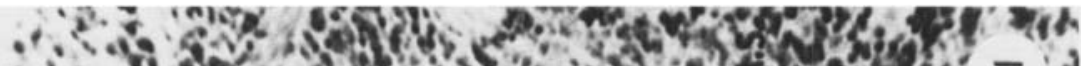

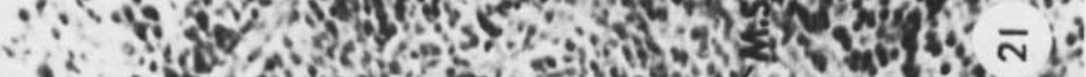

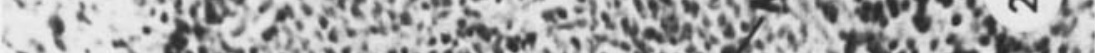

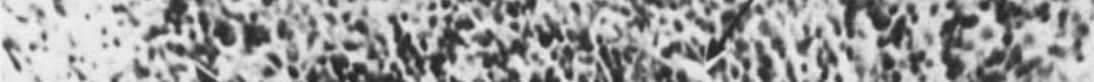

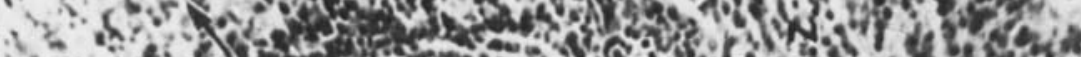

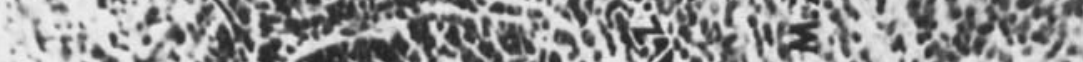
$\therefore$ t. $\therefore$ is

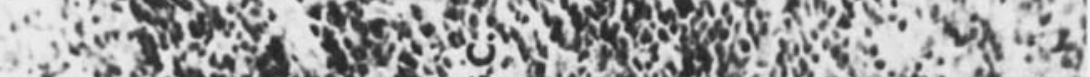

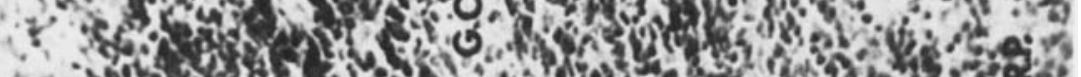

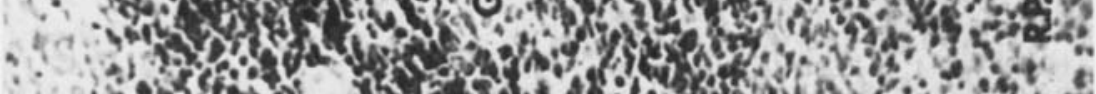

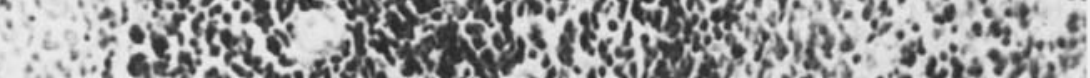

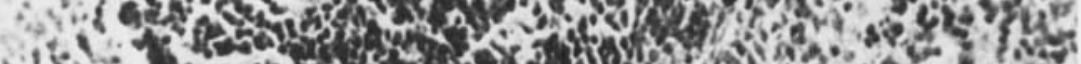

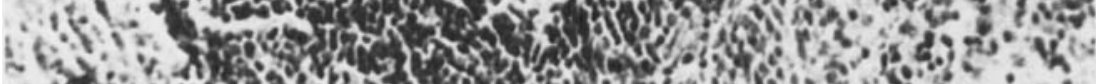

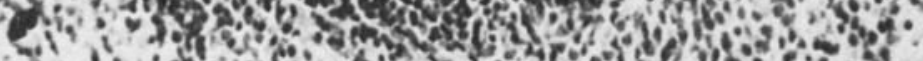

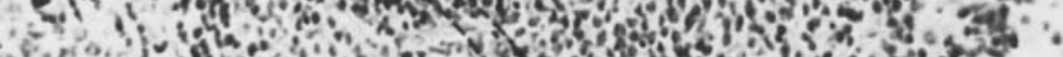

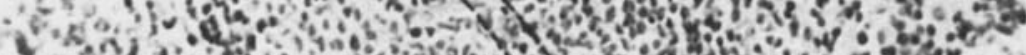

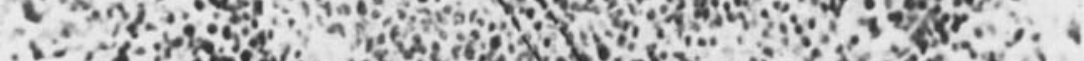

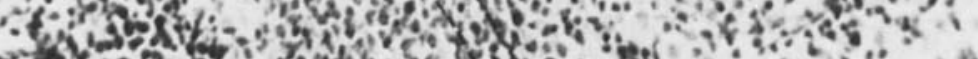

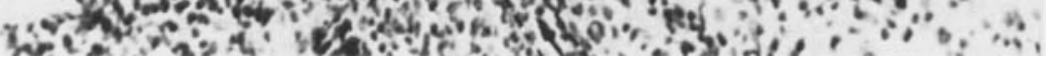


lymphoid parenchyma combine to provide a substantial basis for normal splenic function. It is interesting to note how few of these same factors, singly or in groups, influence the functions of bone marrow as it lies in its protective sheath of bone. The surgical exteriorization of the spleen by itself eliminated several of the physical factors mentioned above and at the same time permitted the application of more manipulatory factors designed for artificial temperature regulations. Allowing for these factor substitutions, the exteriorized spleen appeared to provide the most simplified plateau from which to begin the study. The observations and interpretations of this report crisscross many of the established research interests on the spleen. Some of the observations have no precedence in the literature at the present time; others quite clearly associate the mselves with a variety of current investigative interests on splenic function. It is with the latter that a conventional form of discussion has been entered into.

The results of this study indicate that a number of histological responses occurred when the rat spleen was exteriorized from the body cavity with its blood supply intact and when the splenic temperature was artificially regulated. Foremost of the responses observed was the widespread dissipation from and the destruction of numerous small lymphocytes in the mantle zones and germinal centers in Group I splenic nodules (temperature range of $37^{\circ}$ to $15^{\circ}$ ). In Group 1 spleens, the rate of lymphocyte removal or their debris was proportional to the depth of hypothermia imposed for the three-hour regulated temperature exposure, the highest incidence occurring with the lower temperatures. The resulting reduction in cellularity of the nodules provided a somewhat unique opportunity to visualize and study other temperature-related histologic responses that occurred concomitantly throughout the tissues of the spleen. Of these it was noted that in $37^{\circ}$ and $30^{\circ}$ spleens a sector of the nodule directly opposite the central artery (antiarterial sector) appeared to offer the least restraint for rapid escape of lymphocytes and/or their degeneration products. Sectors immediately adjacent to or more distantly located from this sector of release appeared to be less active in this respect.

Nodules of $26^{\circ}$ Group I spleens were characterized by the formation of a barrier in the form of a cell-pervious dam of hypertrophied marginal metalophils. The dam appeared to restrain the rampant release of cells to the marginal zone and the surrounding red pulp. As a result, the nearly expended mantle zone seen in $30^{\circ}$ spleens (FIG. 7) gave evidence of being reconstituted in $26^{\circ}$ spleens (FIGS. 9 and 10). Terminal degeneration of a majority of the marginal metalophils in $15^{\circ}$ spleens (FIGS. 12,13, and 14) clearly suggested a breakdown in the dam. So few free lymphocytes remained within the nodules at $15^{\circ}$ (FIGS.

FIGURE 21. A Group II $20^{\circ}$ nodule. Reconstitution of the mantle zone (MA.Z.) is less pronounced than in FIGURE 19, of a Group II $26^{\circ}$ nodule. Columns of small lymphocytes (broken-line enclosure) are passing from the sinus area of the nodule into the marginal zone (M.Z.) and surrounding red pulp (R.P.). Release of small lymphocytes is also occurring diffusely from the periarterial white pulp (arrows in upper part of Figure). The lack of retention of small lymphocytes within the nodular area has impaired the formation of a typical mantle zone (MA.Z.). X 100.

FIGURE 22. A higher-power view of the mantle zone (MA.Z.), marginal sinus (M.S.), and marginal zone (M.Z.) of a Group II $20^{\circ}$ nodule. The broken-line enclosure indicates the continuity of a cord of small lymphocytes passing from the mantle zone into the marginal zone. Degeneration of the marginal metalophils (MET.) appears to facilitate the release of small lymphocy tes from the nodule. $x 430$. 


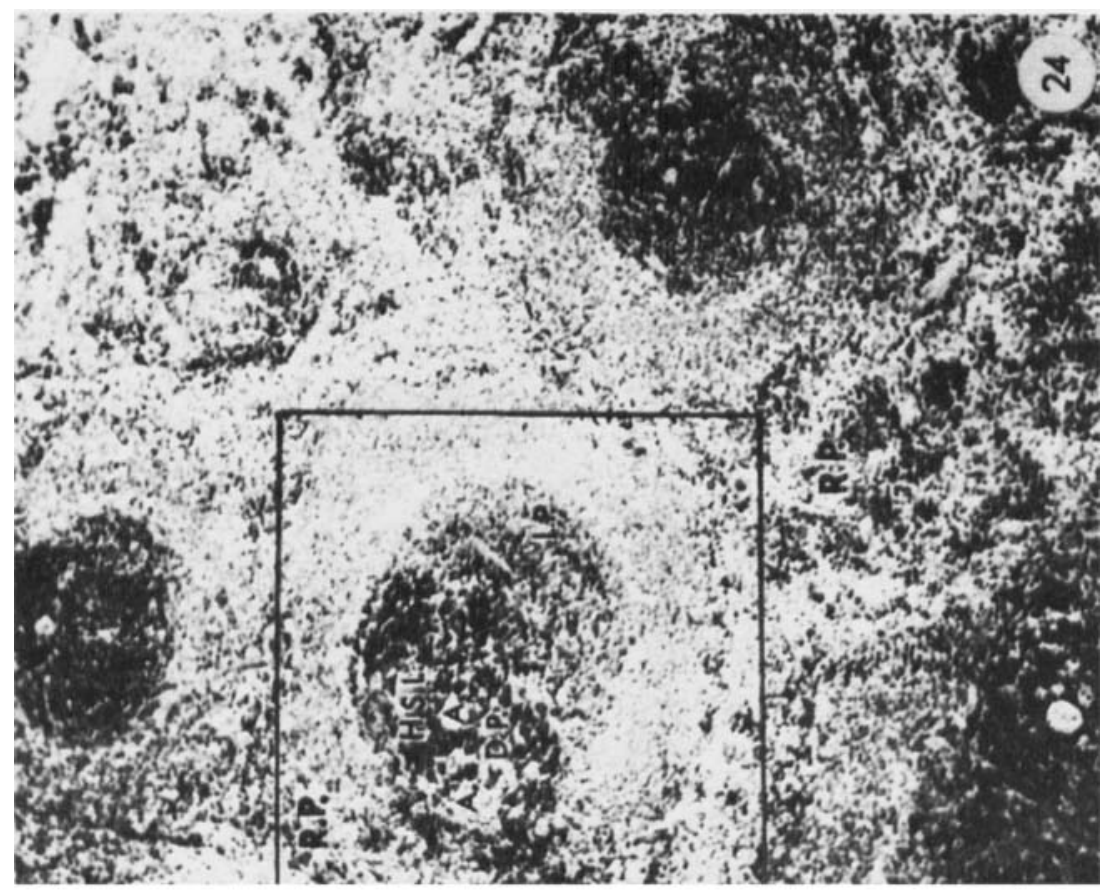

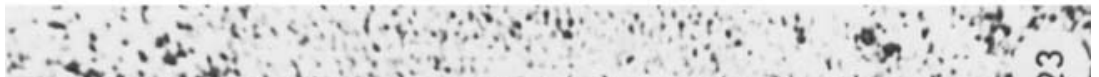
$3 x^{2}+\operatorname{mon}^{2}$

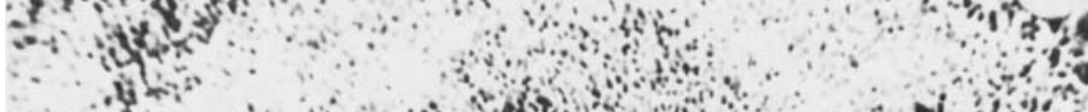

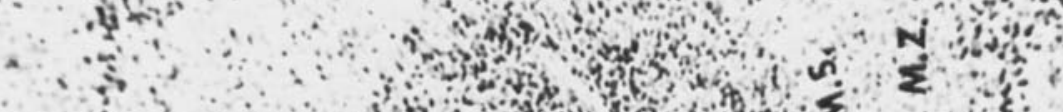
a.

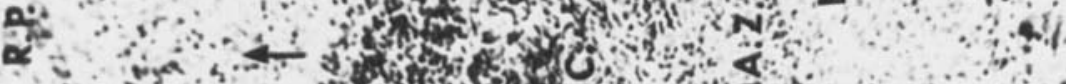

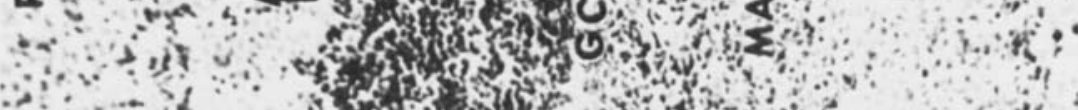
in

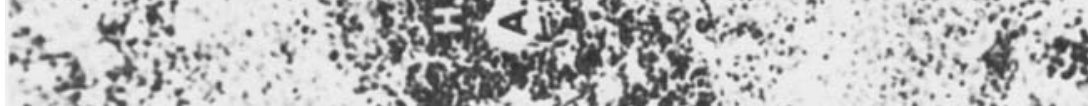

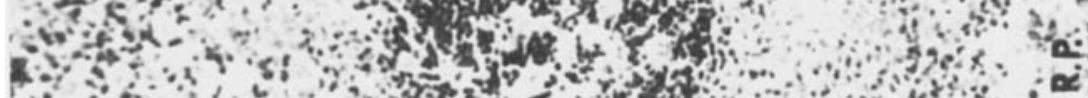

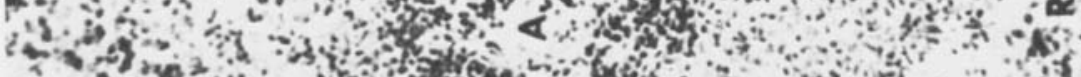

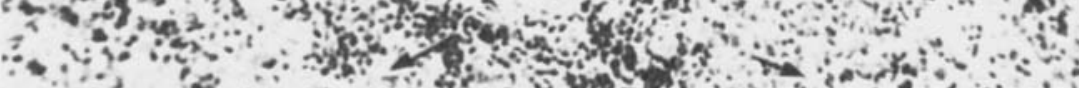

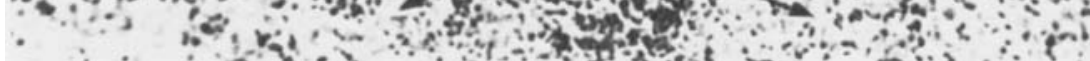


11) that disrupture of the dam was of little consequence in the final dissolution of the nodules.

Histological responses in Group I spleens emphasized the existence of a dichotomy in the cell types comprising the white pulp and nodular tissue. One type, highly mobile and consisting of cordlike masses of small lymphocytes, medium-sized lymphocytes, and large pyroninophilic hemocytoblasts (FIGS. 2, $3,4,5$, and 6) migrated inexorably from the periarterial white pulp and contiguous mantle zone centrally into the germinal centers. In this location, large numbers of the small lymphocytes were observed to undergo pycnosis and subsequent fragmentation. Tingible body macrophages were observed to phagocytize the degeneration products of these cells (FIGS. 3 and 4) throughout the germinal centers. Normal-appearing cells together with pycnotic ones that escaped phagocytosis continued through the nodular tissue to be released in considerable numbers through the thin antiarterial sector (FIGS. 2, 3, and 6). Many of these cells accumulated in the marginal sinus opposite the thin (antiarterial) sector (FIGS. 3 and 6) prior to their passage into and through the marginal zone. Once in the marginal zone, diffuse radial migration of the small lymphocytes or their degeneration products continued as a constant phase into the red pulp (FIGS. 3, 6, 7, and 9). It should also be stated that normal-appearing small lymphocytes, and pycnotic ones as well, migrated in lesser numbers from the periphery of the mantle zone in a similar fashion along the remaining surfaces of the nodule that were more distantly located to the thin sector just described. Cells comprising the cyto-framework of the nodules (undifferentiated stellate reticular cells, marginal metalophils, and macrophages) represented the more stabile members of the cell dichotomy through which the migratory cells passed. Since they were functioning as a quasi-stromal support for the migratory cells, there was no evidence that cells of the cyto-framework contributed to lymphocytopoiesis per se. All forms of the migratory cells, however, were observed to undergo mitosis, especially in the vicinity of the periarterial white pulp and in the germinal centers of $37^{\circ}, 30^{\circ}$, and $26^{\circ}$ spleens. This feature was most prominent at the two higher-temperature levels just cited. No mitotic activity was observed in $15^{\circ}$ specimens.

The selective ability of marginal metalophils to undergo hypertrophy and to develop a highly vacuolated cytoplasm at $26^{\circ}$ is of singular interest. On the basis of nuclear structure alone under normothermic and hypothermic conditions,

FIGURE 23. A Group $1118^{\circ}$ splenic nodule. Numerous hyperchromatic small lymphocytes (H.S.L.) originating from the periarterial (A) lymphatic sleeve have infiltrated the nodular area in an irregular fashion. An organized germinal center (G.C.) and mantle zone (MA.Z.) cannot be identified. Marginal metalophils have degenerated. Only an occasional residue of their former presence could be identified. The marginal sinus is broad and ill-defined and contains only scattered examples of peripheral blood. Large numbers of small lymphocytes are passing or have passed through the marginal zone (M.Z.) to the red pulp (R.P.). Arrows indicate the more obvious exodus of small lymphocytes. $\times 100$.

FIGURE 24. A lower-power view (squared area) of the nodule depicted in FIGURE 23. Several adjacent nodules are visible, enclosing a greater expanse of red pulp. The nodule within the square reveals a dark pole (D.P.) consisting of hyperchromatic small lymphocytes (H.S.L.) in the periarterial (A) lymphatic sleeve. A light pole (L.P.) to the right contains only a minimum of small lymphocytes. The red pulp (R.P.) surrounding each of the nodules indicates a wreath of small lymphocytes that have accomplished their passage from the nodule. $\times 75$. 


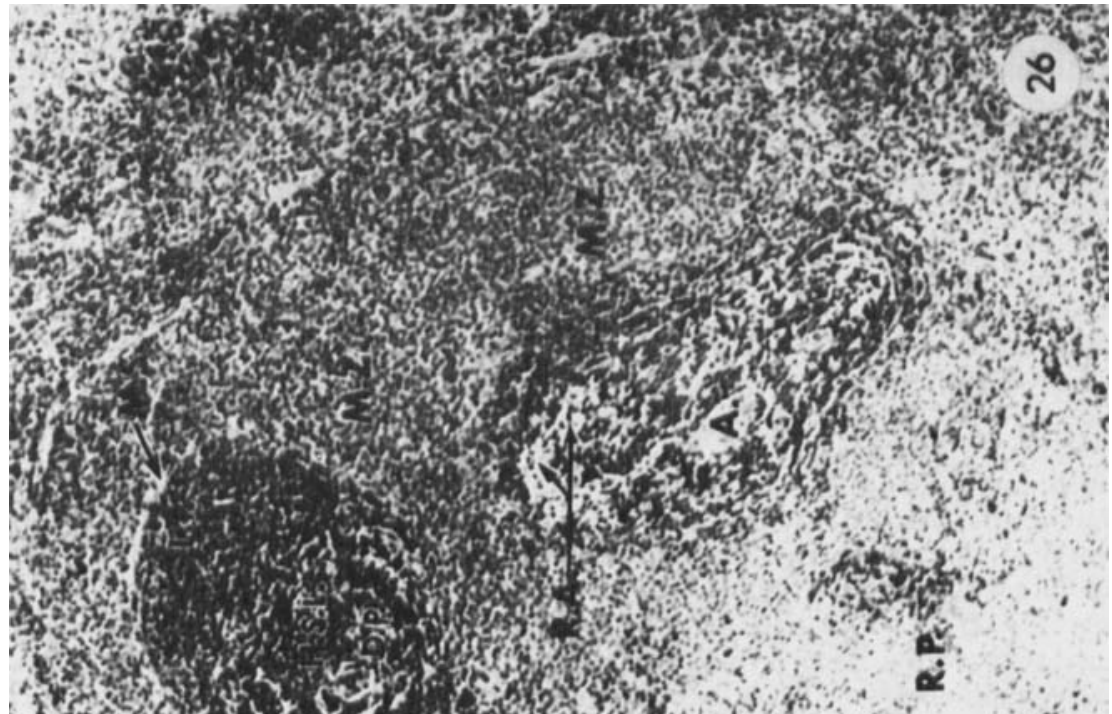

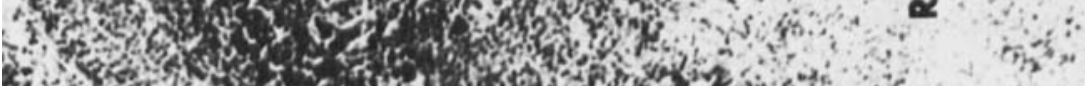

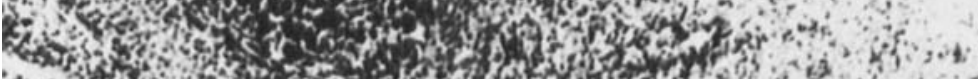

" wo is

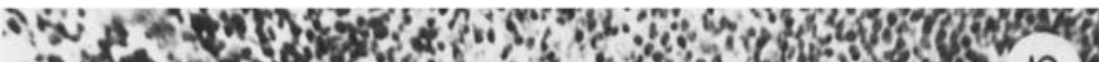

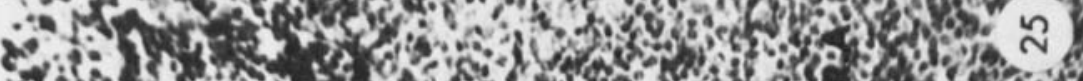

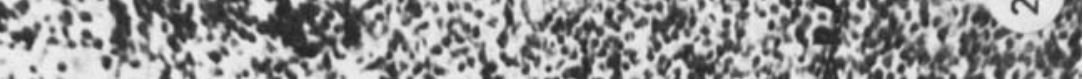

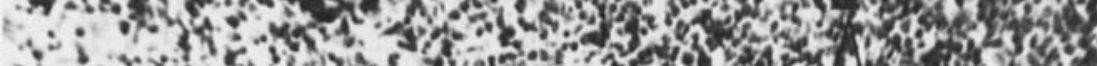
$=3$ - *

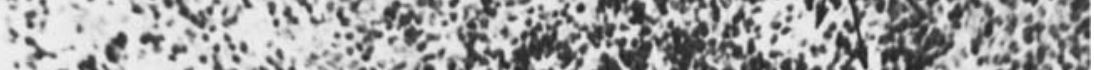

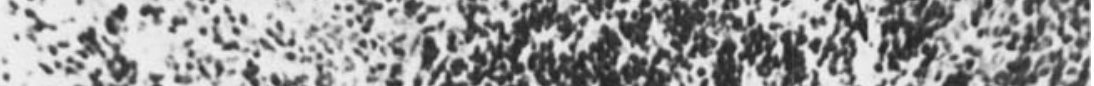

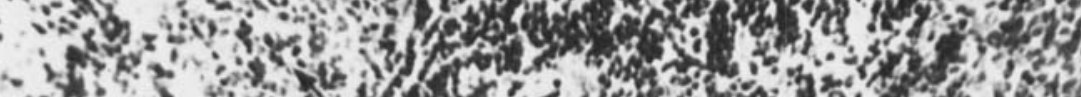

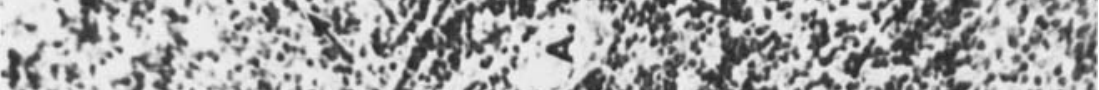

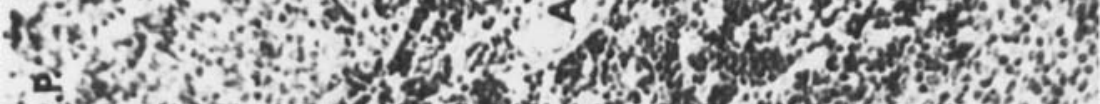
a. 1.7.5.

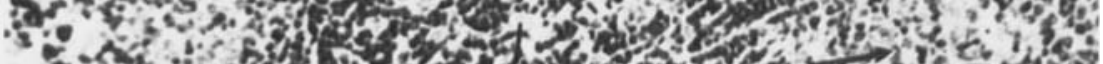

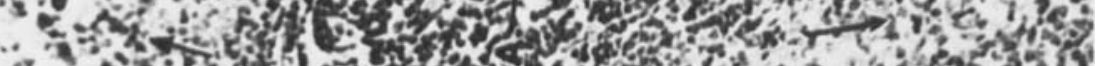

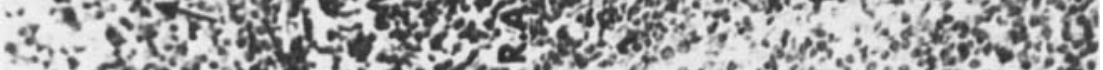

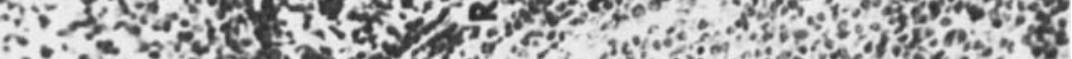
$\therefore$ " $\because 6 * 34$.

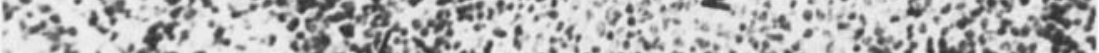

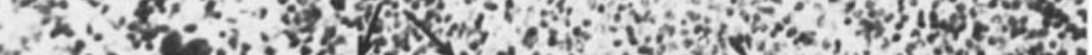

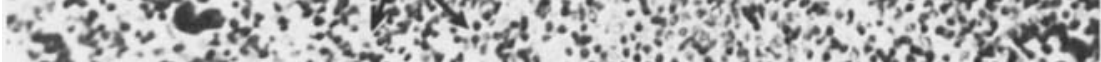
-3.0. 
marginal metalophils revealed a strong similarity to fibroblasts. The pale, relatively achromatic nuclear structure of the metalophils was consistent with that of the fibroblasts located generally in the capsule and trabeculae of the spleen. Moreover, neither fibroblasts nor metalophils were observed to undergo mitosis. The temperature-induced hypertrophy of the marginal metalophils, however, clearly suggested a functional modification that was peculiar to them and distinguished them from typical fibroblasts. Krumbhaar ${ }^{25}$ referred to marginal metalophils as "a young type of fibroblast" and noted their association with the rind of collagenous fibers that surrounded and supported the nodule proper. On the basis of silver impregnation, Marshall $\mathbf{2 8}$ alluded to marginal metalophils as "reticulum cells." Employing Marshall's silver impregnation technique, Snook ${ }^{37}$ graphically revealed the precise distribution of marginal metalophils in relation to the splenic nodule as well as the stellate metalophils located more centrally within the nodule. Snook regarded the latter as fixed reticular cells of the lymphoid tissue. The question arises: Does silver impregnation of metalophils indicate that these cells are macrophages belonging to the reticuloendothelial system? Since the in vitro immunochemical studies of Fishman ${ }^{17}$ and Fishman and Adler, ${ }^{18}$ supportive evidence has accumulated to demonstrate that the presence of macrophages is essential in an incubation medium in order for lymphoid tissue to produce antibodies in the presence of antigen. It has, therefore, become increasingly important in immunological studies to identify the sites in lymphoid nodules where macrophages exist. Marshall ${ }^{28}$ did not consider metalophilia as an equivalent of phagocytic activity, although he believed that certain similarities appeared to exist between the $m$. Snook $^{37}$ injected India ink, chlorazol black $\mathrm{E}$, and saccharated oxide of iron into the tail vein of rats. In ten to fifteen minutes postinjection the injected material was localized in the interstices of the marginal zone. By four to eight hours most of the material was to be found in red pulp macrophages. None of the injected material was found in the marginal metalophils, with the exception of the iron, and this was not uniform. Snook concluded that since marginal metalophils will take up iron under certain conditions, they are real or potential members of the reticuloendothelial system. In this connection it is interesting to note that Hunter ${ }^{24}$ observed only small amounts of titanium dioxide contained in marginal zone phagocytes 15 minutes after injections of 500-, 250-, 125-, and $50-\mathrm{mg}$. doses of this chemically inert material. The predetermined particle size of the $\mathrm{TiO}_{2}$ varied from 0.1 to 1.0 micron, suggesting a particle size of greater dimension than the India ink injected by Snook. ${ }^{37}$ Hunter did not specify the

FIGURE 25. A Group II $14^{\circ}$ spleen (compares to a Group I $15^{\circ}$ spleen). Arrows indicate the release of columns and masses of hyperchromatic small lymphocytes from periarterial (A) white pulp through the marginal zone (M.Z.) into the surrounding red pulp (R.P.). Rarefied areas (R.A.) in the white pulp (indicated by arrows) represent areas of severe lymphocyte depletion resulting from active release. Compare with FIGURE 8 (Group I $30^{\circ}$, in which a more minimal release is occurring. $\times 110$.

FIGURE 26. A Group II $14^{\circ}$ nodule and white pulp. The nodule in the upper left of the figure indicates the continued passage of hyperchromatic small lymphocytes (H.S.L.) from the dark pole (D.P.) or the periarterial sheath (A) into the former light pole (L.P.). Except for the pattern of distribution of the cells, the two poles have been equalized. Compare with FIGURES 23 and 24. Myriads of small lymphocytes in the marginal zone (M.Z.) indicate the diffuse emission of these cells from the nodule. The white pulp nearer the center of the figure further augments the features described for FIGURE 25. $\times 75$. 
origin of the phagocytes but noted that they were located along the inner edge of the marginal zone. Whether he was referring to metalophils as phagocytes or macrophages from one of four sources described by Snook for diseased rats is not clear.

In the study being reported, an unusual, unobstructed view of marginal metalophils could be achieved owing to the reduced cellularity of the nodules. In FIGURES 9, 10, and 14, the precise location of these large cells may be identified. FIGURES 9 and 10 are from a $26^{\circ}$ Group I spleen. It can be noted in FIGURE 10 that the marginal metalophils are large and rounded and contain cytoplasmic vacuoles of uniform size. The nuclei are pale, ovoid in shape, and similar to those of typical fibroblasts. It may also be noted in FIGURE 10 that the marginal zone consists of only scattered cords of cells, thus allowing free communication of blood from the marginal sinus through the marginal zone to the red pulp. Concurrent with this increased conduction of blood, the vacuoles of the marginal metalophils were observed to contain an amber-colored material suggestive of a hemoglobin lysate. Although numerous small particles of nuclear debris may be seen throughout the nodule (FIG. 10), such particulate matter was never taken up by the marginal metalophils. The several vacuolated marginal metalophils seen in FIGURE 14 (Group I $15^{\circ}$ spleen) all contained the same lysate material seen in $26^{\circ}$ spleens. It appears that some type of relationship exists between marginal metalophil hypertrophy and the imbibition of materials in solution. Since the imbibed materials occupy vacuoles that are uniform in size and equally spaced within the cytoplasm, with no tendency to vary from this pattern, it is difficult to conceive that such cells are typical macrophages. As stated before, these cells do not take in visible particulate matter, nor do they appear to have the ability to segregate materials in vacuoles of varying sizes. The upper part of FIGURE 14 is the marginal zone. An indefinite marginal sinus communicates with blood spaces between the lymphoid cords of cells in the marginal zone similar to that seen in FIGURE 10 (26 spleen).

Although the term "migration" has been used in the present study to describe the passage of lymphocytes from the splenic nodules, the term "flushed" would seem to be more accurate. The term "migration" implies active participation on the part of the cells, but since lymphocyte debris departed from the nodules with the same facility as did normal cells, the use of the term must be viewed with reservation. Even allowing for species differences, arterial blood pressure appears to be of primary functional importance in the regulation of the plasma and corpuscular fractions of the blood delivered to the finest terminal arterioles of the white and red pulp. Regional and functional differences in these terminals appear to form a partial basis for specificity of functions peculiar to the spleen. $2,26,32,38-40,44$ While some small arterioles of the nodules selectively permit plasma transudation in such copious quantities that corpuscular concentration occurs intravascularly, others devoid of cells seem to routinely conduct plasma alone. Extravascular plasma, indeed, appears to function as an "inflating" medium for the nodules. Even with the severe loss of cells seen in Group $I 15^{\circ}$ spleens (FIG. 11), the nodule maintains its rounded contour. It is rare to find misshapen nodules even under experimental conditions. In a Group II $26^{\circ}$ spleen (FIG. 19) an irregular contour has appeared at the periphery of the nodule, but in this instance unequal growth of the regenerating mantle layer appears to be the causative factor of the irregularity observed.

Hydrodynamic principles suggest the possibility of an increased hydrostatic pressure generally throughout the white pulp and especially within the nodules. The presence of considerable plasma transudation, an intense compaction of 
relatively small cells, and a pressure-relief membrane, consisting of a fine web of collagenous and argyrophil fibers (Sainte-Marie and $\mathrm{Sin}^{34}$ ), all contribute to an impoundment that would elevate hydrostatic pressure. Free cells (ly mphocytes, hemocytoblasts, and plasma cells) in the interstices of the white pulp could be expected to respond to a flow or flush pattern of the plasma as the latter seeks release to a lower hydrostatic pressure. Depending on the relative porosity of the pressure-relief membrane, the movement of plasma and cells could be rapid or very slow. The inclusion of marginal metalophils along the relief membrane could be expected to modify the porosity of the membrane additionally. The more expansible red pulp together with its venous drainage clearly suggests an area of the spleen that is under considerably less hydrostatic pressure. All of the other physical factors (cited in the first paragraph of this discussion) seem to act, in one way or another, as augmenters of the concept just described.

A related but highly controversial layer of the red pulp, the perifollicular envelope or marginal zone, lies in an intermediate position between the nodules and red pulp proper. In the rat, all of the corpuscular elements derived from the marginal sinus and from some of the nodular arterioles, as well as from arterioles derived from the penicillar complex, are intercepted and delayed in this envelope or zone before reaching the red pulp proper. In this sense, the marginal zone must be considered as a final pressure-relief device that influences the egress of mobile cells and plasma from the nodules. In normal normothermic spleens the marginal zone consists of irregularly radiating cords of pale cells with small irregular spaces between individual cells and adjacent cords of cells. According to Krumbhaar ${ }^{25}$ and Baillif ${ }^{3}$ the cells resemble medium-sized lymphocytes. Baillif further noted that the cells remain physically associated with one another, as would be true of mesenchyme. Snook ${ }^{37-40}$ has demonstrated that the cells comprising the marginal zone are supported by fine argyrophil fibers in the form of a delicate net. No sinuses exist in the marginal zone; blood sequestered in the interstices of the marginal zone parenchyma is presumed to be lysed or altered, thus representing one of the more important functions of the spleen. At the junction of the marginal zone with the red pulp, the cords of marginal zone parenchyma separate from one another, and, as a consequence, red pulp cords may be seen to penetrate into the marginal zone for variable distances. Krumbhaar called attention to the labile character of the marginal zone under experimental conditions. He noted a marked reduction in thickness of the marginal zone in rats subjected to hemopoietic poisons and its return to a normal condition postexperimentally. It is also a common observation in normal spleens to find prominent compression of marginal zones where two closely adjacent nodules impinge on one another. In such instances the intervening red pulp is frequently reduced to a compact, narrow band.

In hypothermic spleens, progressive marginal zone rarefaction was observed to occur as a correlate of temperature depression. The first signs of rarefaction occurred in Group $130^{\circ}$ spleens (FIG. 7). Widened interstices between individual cells and cords of cells were evident. Except for bands of migrating pycnotic lymphocytes, the marginal zone consisted primarily of marginal zone parenchyma. An accumulated mass of pycnotic lymphocytes at the junction of the marginal zone with the red pulp is indicative of a previous surge of cells through the marginal zone. In Group I $26^{\circ}$ and $15^{\circ}$ spleens (FIGS. 10, 11, 12, 13, and

14) progressive rarefaction has continued to a point where the marginal zone freely conducts blood from the marginal sinus to the red pulp. Once this circumstance obtains, the marginal zone ceases to act as a final pressure relief 
and functions instead as a modified zone of the red pulp. Red pulp macrophages extended deeply into the former marginal zone, many of them revealing hemosiderin deposits. It is noteworthy that in Group II spleens in which partial reconstitution of the marginal zone occurred $\left(26^{\circ}, 20^{\circ}, 18^{\circ}\right.$, and $14^{\circ}$ spleens), occasional he mosiderin-laden macrophages of the red pulp type were observed to remain within the newly forming marginal zone. This observation may provide an explanation why Krumbhaar ${ }^{25}$ considered the marginal zone a principal site of hemosiderin accumulation in his experimental animals. It is quite likely that major depletion of the marginal zone followed by subsequent reconstitution produces a similar but not identical marginal zone parenchyma. The observations cited on the marginal zone of hypothermic spleens may apply restrictively to the experimental procedures utilized. The histological features observed, however, may at the same time provide additional insight in to similar events occurring under a variety of experimental conditions as well as under normal physiologic conditions.

The marginal zone parenchyma has also generated considerable interest with regard to its hemopoietic significance. Krumbhaar ${ }^{25}$, in addition to noting lymphocyte-forming malpighian follicles, described "two rather distinct hemopoietic areas" in the rat spleen. These were small foci scattered throughout the red pulp and perifollicular areas. He noted hemopoietic cells of several types, excluding megakaryocytes, interspersed in the perifollicular areas of rat spleens recovering from hemopoietic poison. He was of the opinion that, at least in some instances, concentrated foci of these cells suggested local hemopoiesis. Baillif ${ }^{3}$ also observed erythropoiesis and phagocytogenesis in the white pulp halo of his thorium-dioxide-treated rats. Weiss ${ }^{43}$ believed that large lymphocytes and other free cells of the marginal zone correspond to analogous cells found in the germinal centers. He noted that in the rat and rabbit, germinal centers are not well developed and in these species the marginal zone may be the functional equivalent of the germinal centers. More recently, Petterson and colleagues, ${ }^{31}$ studying the primary and secondary immune responses in the rat spleen, concluded that the plasma cell precursors are located in the marginal zones. They stated that, in less than one day after injection of antigen (typhoid and $E$. coli), marginal zone cells migrated into the nodules. Mitosis of these cells and their subsequent differentiation into hemocytoblasts were observed to occur in the presence of nodular macrophages. The hemocytoblasts were then believed to migrate from the germinal centers across the marginal zone again into the red pulp, where they differentiated into mature plasma cells in the company of red pulp macrophages. Differing only in degree-time relationship, they noted the following events after primary injection of $E$. coli and typhoid-paratyphoid vaccine: the marginal zone narrowed to about one-fourth its normal width several hours after either primary or secondary injections, and the nodules enlarged proportionately. During the narrowing stage there were of ten significant numbers of macrophages within the zone. By the fourth day, the marginal zone returned to its normal width, $80 \mu$, the latter being true for both primary and secondary injections.

Several points of interest or comparisons may be made between the results of Petterson and co-workers ${ }^{31}$ and the effects of artificial temperature regulation on the same organ and species. It should be emphasized that no antigen was injected or otherwise employed in the study being reported. All of the animals used were normal, healthy, and in a natural state of immunity typical of specimens found in a carefully managed animal-care facility. Incursions of 
pyroninophilic hemocytoblasts into the germinal centers from the periarterial white pulp and mantle zone were commonplace in $37^{\circ}$ to $26^{\circ}$ Group I animals. Although many of these prominent cells were associated with nodular macrophages, some were also constantly being released from the nodules in to the marginal zone, especially through the thin antiarterial sector. Plasma pressure gradients appeared to enhance their progress through the nodule, and in general they tended to follow the same course of release as the myriads of small lymphocytes that accompanied them. Unless a counter force greater than that of the plasma pressure can be conceived, it is difficult to accept the hypothesis of Petterson and associates that marginal zone cells, in fact, invade the nodules from their peripheral marginal zone location. Staining similarities of marginal zone cells with those of nodular hemocytoblasts are, at best, only suggestive evidence of the origin of the former. The dissipation or narrowing of the marginal zone observed by Petterson and associates would have more significance if they described how this occurred rather than simply stated that it did occur. If by inference the increase in size of the nodule is to suggest the massive loss of marginal zone cells to the nodule several hours after primary and secondary injections, then the problem of plasma pressure gradients becomes compounded. This concept would be inconsonant with the injection studies of Snook ${ }^{37}$ and the effects of hypothermia. In severe local hypothermia of the spleen (FIGS. 11 and 13) a substrate of cells appeared in the depleted mantle zone (depleted of small lymphocytes) markedly resembling the cells that comprise the marginal zone. Inundated by masses of small lymphocytes in the normothermic nodule, this cellular substrate of modified, medium-sized lymphocytes is rarely visible. The cells are probably a constant feature of the mantle zone and provide structural stability to it. Under the influence of hypothermia, the most peripheral of the marginal zone cells were observed to lose their pyroninophilia and separate from one another. In so doing, individual cells were lost amongst the cells of the encroaching red pulp cords. Peripheral attrition of the marginal zone and its replacement by red pulp was observed to vary according to the depth of hypothermia utilized. The mode of reconstitution of the marginal zone was less clear. Waves of irregular concentric increment of cells beginning adjacent to the marginal sinus were observed to progressively compact a regenerating marginal zone (FIGS. 19 and 23). An irregular void usually intervened between the first row of cellular compaction and the marginal sinus. Sparse cellular areas persisted as well in the otherwise highly cellular compacted areas of the marginal zone. Medium-sized lymphocytes derived from the mantle zone and from marginal zone arterioles appeared to be the major source of the cells during the reconstruction phase.

In all Group II animals the return of the spleen to its normal environment (reperitonealized for a period of 72 hours) resulted in a marked recovery of the mantle zone and, in some instances, a partial recovery of the marginal zone. Compare the $37^{\circ}, 30^{\circ}$, and $26^{\circ}$ Group II spleens with their temperatureequivalent counterpart in Group I spleens. Very few traces of pycnosis and degeneration debris remain in Group II nodules, and the egress of normal small lymphocytes into the marginal zone appears to be occurring at a more normal rate. Group II spleens subjected to severe hypothermia $\left(20^{\circ}, 18^{\circ}\right.$, and $\left.14^{\circ}\right)$ also show recovery of the mantle zone (FIGS, 21-24 and 26). With severe hypothermia, regeneration of the mantle zone can be seen to begin in the periarterial white pulp and to extend from this location around the margin of the nodule in an irregular fashion (FIGS. 21 and 24). Temperature damage to 
the nodular cytofibrous framework appears to have occurred in $14^{\circ}$ nodules and to a lesser extent in $18^{\circ}$ spleens. This observation is based on the apparent inability of $14^{\circ}$ spleens to form a typically peripheral mantle zone; instead, there occurs a diffuse infiltration of the entire nodule with small lymphocytes in the absence of a definable mantle zone and a germinal center (FIGS. 23 and 26). The former nodule, as a consequence, resembles modified white pulp. The unrestrained emission in such nodules and contiguous white pulp of large numbers of small lymphocytes into and through the marginal zone further suggests an ineffectual pressure relief mechanism with regard to the flushing pressure of plasma and the cells it contains (FIGS. 23, 25, and 26).

It should be emphasized that pyroninophilic hemocytoblasts were commonly observed among the small lymphocytes in the periarterial white pulp of all Group II spleens. Both the hemocy toblasts and small lymphocytes underwent mitosis, but never at a rate that would suggest a substantial increase in cell numbers. The incidence of mitoses was very low to absent in severe hypothermia, but this did not prevent the appearance of a substantial stock of cells in the immediate vicinity of the white pulp arterioles. A review of the figures of Group I and II nodules will reveal varying numbers of hyperchromatic lymphocytes circumscribing the branches of the central artery. Han and colleagues $^{22}$ call attention in their Figure 9 to a similar observation in their endotoxin treated rats. They could have made the same observation from their Figure 8 of a control rat. Baillif ${ }^{3}$ also observed a stock of pycnotic microlymphocytes in the lymphatic sheaths in white pulp arteries in his thorotrast-injected rats. Fliedner and associates, ${ }^{19}$ on the basis of $H^{3}$ TDR labeling of the rat spleen, left open the possibility that mantle zone cells may act as a focus of new germinal center development. They believed, however, that no genetic relation exists between the rapidly proliferating cells in the germinal centers and the cells in the surrounding mantle zone. Their data suggested that mantle zone cells represent part of a "storage pool" of slowly proliferating lymphocytes under steady-state conditions. In the present study, evidence suggests that columnlike masses press into the germinal centers from the mantle zone and thereby provide a significant proportion of the dividing and reacting cells. The possibility always exists that other cells, through diapedesis, enter the germinal centers via nodular arteries. The concept of Fliedner and colleagues of the mantle zone as a "storage pool" suggests a degree of inherent stability. Spleens subjected to local hypothermia seem to indicate that the cellular composition of the mantle zone is very labile, the turnover of cells being continuous.

The site of origin of the small lymphocytes, which were a constant feature of the periarterial lymphatic sheaths and appeared to represent a significant regenerative potential to the morphologic reconstitution of the temperaturealtered nodules, could not be determined in the study being reported. Weiss ${ }^{43}$ considered the lymphocyte infiltrations of the periarterial sheaths to be thymic-dependent. He considered lymphatic follicles as local nodular thickenings occurring within or directly alongside the sheaths. He believed that the periarterial white pulp has the capacity both to selectively remove ly mphocytes from the blood and to support mitotic divisions among resident lymphocytes. The fine, radially oriented branches of the central artery were observed to contain mural apertures. These observations appear to agree with or support the findings in the present study. Ford and Gowans, ${ }^{20}$ by means of radioautographic studies, however, have demonstrated that many long-lived small 
lymphocytes recirculate continuously between the blood and the periarteriolar lymphoid sheaths in the spleen. The thymidine- $\mathrm{H}^{3}$ DNA-labeling studies of Everett and Tyler (Caffrey) ${ }^{10}$ indicate that some small lymphocytes have a short life span while others live for a very long period. In the present study it was a matter of some interest to note that $37^{\circ}, 30^{\circ}$, and $26^{\circ}$ nodules of Group 1 spleens always revealed normal-appearing small lymphocytes, which traversed the various zones of the nodule unscathed by temperature regulation while many others underwent total destruction. In future study it would be interesting to ascertain whether a correlation exists between the two categories of small lymphocytes described by Everett and colleagues and those observed in the study reported here.

A projection of one frequent observation in the present study should be made, since its functional interpretation appears to have a bearing not only on temperature-regulated spleens but also on spleens assumed to be normothermic. The appearance of fine to more obvious cracklike separations in lymphoid tissues of the spleen is commonplace in published figures throughout the literature. In some instances, the cracklike configurations may be due to faulty tissue technique; in many others this may not be true. Preliminary studies of splenic leucocytes in isotonic solution held at slightly subnormal temperatures revealed marked reduction in cell volume. A marked reduction in cell volume was also observed with subnormal temperatures when plasma was used. In the latter, some of the contracted cells were also observed to agglutinate. This is to suggest that, even with minimal temperature variations, the free cells of the splenic white pulp and nodules tend to contract and in some instances agglutinate within the organ. A resulting volume imbalance between cells and plasma appears to be contributory to the cracklike configurations in question. Factors other than temperature may also be operative. The flushing effect referred to throughout this text appears to be related to an increase in plasma volume in the presence of a reduction in cell volume. Any laparotomy performed at room temperature results in some degree of hypothermia to the organs exposed. The greater the exposure in relation to time and the greater the cooling effect through evaporation of surface moisture, the more hypothermic the organ(s) become. In connection with the foregoing, the use of anesthesia is known to depress body temperature two or more degrees. Studying the effects of temperature variation in living exteriorized mouse spleens by direct observation with transillumination, Peck and Hoerr ${ }^{30}$ observed an increased storage of blood in the red pulp when the temperature of the Ringer's bath solution was depressed and a nearly continuous flow when the temperature was elevated. They also observed volume changes in the red and white zones of the organ as the temperature was varied. A strong initial contraction of the spleen was observed to occur when the bath temperature was suddenly raised or lowered. The responses cited by Peck and Hoerr emphasize the importance of the temperature factor in studies on the spleen, in view of the fact that the responses cited were observed to occur within a temperature span of approximately $3^{\circ}$ above or below $37.5^{\circ} \mathrm{C}$.

The full significance of the effect of temperature regulation on splenic function cannot be appreciated unless acceptable methods of standardization are developed. The present study represents one effort in this direction. The thermal regulatory apparatus, as conceived, has provided accurate temperature control to organs in two independent studies, the one being reported here and another on the effects of hypothermia on pancreatic exocrine secretion. ${ }^{45}$ The apparatus is 
versatile in its applications, being limited only by the ability of the operator to conceive and construct a suitable thermal end capsule for the requirements of the anticipated study.

\section{SUMMARY}

In order to determine the specific effects of temperature variation on the histological response of the nodular tissue and white pulp of the rat spleen, a thermal regulatory apparatus was designed and constructed. The apparatus, consisting of a pump-driven cold and heat exchanger, was linked electronically to and controlled by a Yellow Springs Thermistempt temperature controller by way of several sensor probes. A coolant or cryogen propelled from the pump to a thermal end-capsule in which the exteriorized spleen was inserted served to offect temperature transfer. Core temperature of the rat was maintained by a thermostatically heated table. A 12-channel Yellow Springs Telethermometer recorded all required temperatures of both the apparatus and the experimental animal. The apparatus maintained a constant predetermined temoerature to the exteriorized spleen within $\pm 0.1^{\circ} \mathrm{C}$.

Two groups of animals, the spleens of which were subjected to temperature control, were studied and compared. Spleens of one group were exposed to either $37^{\circ}, 30^{\circ}, 26^{\circ}$, or $15^{\circ}$ for a three-hour period, after which splenectomy was performed. Spleens of a second group were exposed to either $37^{\circ}, 30^{\circ}, 26^{\circ}$, $20^{\circ}, 18^{\circ}$, or $14^{\circ}$ for a corresponding time but were reperitonealized for three days before splenectomy was performed. In the first group, numerous small lymphocytes underwent destruction or were flushed from the nodules commensurate with temperature depression. In the reperitonealized group (Group II), significant reconstitution of the nodules occurred except at the two lowest temperature exposures.

Marginal metalophils hypertrophied at $26^{\circ}$ in both groups and formed a semipervious dam against the continued emission of lymphocytes or their debris from the nodule. A concomitant disrupture of the marginal zone occurred in Group I spleens, revealing a continuous conduction of blood from the marginal sinus to the red pulp. This feature was less evident in Group II spleens. Hydrostatic factors and changes in cell volume appeared to be altered by marked decrements in temperature, thereby forming a basis for the effusion of plasma and cells from the nodules.

Hyperchromatic small lymphocytes not only persisted in the periarterial white pulp in both groups at all temperature decrements but were observed to increase in number in Group II spleens. Evidence suggests that the increase of small lymphocytes, just cited, is accomplished by migration of cells from the smaller blood vessels of the region. Mitotic activity in the periarterial white pulp of Group II spleens did not appear to materially influence the increase in cell numbers.

\section{CONCLUSIONS}

1. The thermal regulatory apparatus as described, has consistently and accurately delivered artificial temperature control to the exteriorized rat spleen within a range of $37^{\circ}$ to $14^{\circ} \mathrm{C}$. Although not utilized in the study being reported, it is equally capable of delivery of artificial temperature control over a broad range above $37^{\circ}$. 
2. Depending upon the level of temperature depression utilized during a three-hour exposure, vast numbers of small lymphocytes within the nodules were destroyed and, together with normal cells, were flushed from the nodules into the red pulp. This suggests that a marked temperature sensitivity exists in many of the small lymphocytes of the rat spleen.

3. Reference to the flushing effect in conclusion 2 further suggests that artificial temperature depression of the exteriorized spleen results in alterations of hydrostatic pressure within the nodules and a greater porosity of the pressure-relief membrane surrounding each nodule. Hypertrophy of marginal metalophils or their destruction appears to further modify the egress of cells from the nodules. The marginal zone also appears to function as a restraint to the flushing effect. In this sense it may be considered as a final pressure-relief membrane.

4. The greatest egress of cells from the nodule appears to occur in the antiarterial sector.

5. The significant events of conclusions 2 through 4 are readily observable by means of ordinary serially sectioned preparations of the spleen.

6. Spleens that were reperitonealized for three days after exposure to temperature depressions of $37^{\circ}, 30^{\circ}$, and $26^{\circ}$ (Group II) reveal marked recovery or reconstitution of nodular structure.

7. Artificial temperature depression as described in this study opens the door to many new possibilities for understanding the immune responses of the mammalian spleen. This is to suggest that the reacting cells of a primary or secondary immune response may be reduced, destroyed, or otherwise altered within the spleen at any point in the response and the effects of such an action interpreted by reperitonealizing the spleen for a variable period of time.

\section{ACKNOWLEDGMENTS}

The author wishes to express gratitude to Prof. William L. Brudon for illustrations and advice in photography and to Prof. Theodore C. Kramer and the late Prof. William W. Steinberger for helpful advice in mechanics and electronic construction of the thermal regulatory apparatus used in this study.

\section{REFERENCES}

1. ALLEN, F. M. 1937. Local asphyxia and temperature change in relation to gangrene and other surgical problems. Trans. Ass. Amer. Physicians 52: 189-204.

2. ALTSCHUL, R. \& F. A. HUMMASON. 1947. Minimal vascular injections of the spleen. Anat. Rec. 97: 259-264.

3. BAILLIF, R. N. 1953. Splenic reactions to colloidal thorium dioxide in the albino rat. Amer. J. Anat. 92: 55-115.

4. BARNARD, C. N. 1956. Hypothermia: a method of intragastric cooling. Brit. J. Surg. 44: 296-298.

5. BLADES, B. \& C. PIERPONT. 1954. Simple method for inducing hypothermia. Ann. Surg. 140: 557-561.

6. BURCH, B. H., D. W. TRAPHAGEN, M. J. FOLKMAN, A. ROSENBAUM \& E. G. MUELLER. 1954. Temporary aortic occlusion in abdominal surgery. Surgery 35: 684-689.

7. CROSSMAN, L. W., W. F. RUGGIERO, V. HURLEY \& F. M. ALLEN. 1942. Reduced temperatures in surgery: Amputations for peripheral vascular disease. Arch. Surg. 44: 139-156. 
8. DELORME, E. J. 1952. Experimental cooling of the blood stream. Lancet 2: 914-915.

9. DOWNEY, J. A. 1964. Physiologic effects of heat and cold. J. Amer. Phys. Ther. Ass. 44: 713-717.

10. EVERETT, N. B. \& R. W. TYLER (CAFFREY). 1967. Lymphocytopoiesis in the thymus and other tissues: functional implications. In International Review of Cytology. G. H. Bourne, J. F. Danielli \& K. Jeon, Eds.: 205-237. Academic Press. New York, N.Y.

11. FAY, T. 1959. Early experience with local and generalized refrigeration of the human brain. J. Neurosurg. 16: 239-260.

17. FISHMAN, M. 1961. Antibody formation in vitro. J. Exper. Med. 114: 837-856.

18. FISHMAN, M. \& F. F. ADLER. 1963. Antibody synthesis in $x$-irradiated recipients of diffusion chambers containing nucleic acid derived from macrophages incubated with antigen. J. Exper. Med. 117: 595-602.

19. FLIEDNER, T. M., M. KESSE, E. P. CONKITE \& J. S. ROBERTSON. 1964. Cell proliferation in the germinal centers of the rat spleen. Ann. N.Y. Acad. Sci. 113: 578-594.

20. FORD, W. L. \& J. L. Gowans. 1969. The traffic of lymphocy tes. Sem. Hemat. 6: 67-83.

21. GIBBONS, W. D. 1966. Gastric hypothermia (Wangensteen): A preliminary report on cooling and freezing with the use of a new machine. Med. J. Aust. 2: 220-224.

22. HAN, S. S., A. G. JOHNSON \& I. H. HAN. 1965. The antibody response in the rat. I. A histometric study of the rat spleen following a single injection of bovine gamma globulin with and without endotoxin. J. Infect. Dis. 115: 149-158.

23. HERZFELD, C. M., Ed. 1963. Temperature: Its measurement and control in Science and Industry, Part 3, Biology and Medicine: 1-683.

24. HUNTER, R. L. 1966. Two patterns of splenic phagocytosis. New Physician 15: 111-119.

25. KRUMBHAAR, E. B. 1948. A hemopoietic perifollicular envelope in the rat spleen. Blood 3: 953-959.

26. MACNEAL, W. J., S. OTANI \& M. B. PATTERSON. 1927. The finer vascular patterns of the spleen. Amer. J. Path. 3: 111-122.

27. MCINTYRE, J. A. \& R. BRINDIS. 1966. Gastric secretory changes resulting from gastric hy pothermia. Brit. J. Surg. 53: 439-445.

28. MARSHALL, A. H. E. 1956. An Outline of the Cytology and Pathology of the Reticular Tissue. Charles C. Thomas. Springfield, Ill.

29. NABSETH, D. C., R. L. GOODALE \& A. E. REIF. 1960. Studies on the effect of intragastric cooling on acute experimental pancreatitis. Surgery 47: 542-547.

30. PECK, H. M. \& N. L. HOERR. 1951. The effect of environmental temperature changes on the circulation of the mouse spleen. Anat. Rec. 109: 479-493.

31. PETTERSON, J. C., D. F. BORGEN \& K. C. GRAUPNER. 1967. A morphological and histochemical study of the primary and secondary immune responses in the rat spleen. Amer. J. Anat. 121: 305-318.

32. ROBINSON, W. L. 1926. The vascular mechanism of the spleen. Amer. J. Path. 2: 341-356.

33. ROSS, D. N. 1954. Hypothermia: Part 1. A technique of blood stream cooling. Guy. Hosp. Rep. 103: 97-115.

34. SAINTE-MARIE, G. \& Y. M. SIN. 1968. Reticular fiber pattern in the rat spleen. Rev. Can. Biol.1. 27 (3): 273-275.

35. SCHWARZ, M. R., CH. 1969. Symposium on the life history of lymphocytes. Anat. Rec. 165 (1): 105-126.

36. SIZER, I. W. 1943. Effects of temperature on enzyme kinetics. Advances Enzym. 3: $55 \cdot 62$.

37. SNOOK, T. 1964. Studies on the perifollicular region of the rat spleen. Anat. Rec. 148: 149-159.

38. SNOOK, T. 1944. The guinea pig spleen. Study on the structure and connections of the venous sinuses. Anat. Rec. 89: 413-427.

39. SNOOK, T. 1950. A comparative study of the vascular arrangements in mammalian spleens. Amer. J. Anat. 87: 31-78. 
40. SNOOK, T. 1958. The histology of vascular terminations in the rabbit spleen. Anat. Rec. 130: 711-730.

41. WANGENSTEEN, O. H., H. D. ROOT, C. B. JENSON, K. IMAMOGLU \& P. A SALMON. 1958. Depression of gastric secretion and digestion by gastric hypothermia: Its clinical use in massive hematemesis. Surgery 44: 265-274.

42. WANGENSTEEN, S. L. \& W. H. SMITH. 1960. A gastric hypothermic machine. Surgery 47: 924-928.

43. WEISS, L. 1964. The white pulp of the spleen: The relationship of arterial vessels, reticulum and free cells in the periarterial lymphatic sheath. Bull. Johns Hopkins Hosp. 115: 99-173.

44. MACNEAL, W. J. 1929. The circulation of blood through the spleen pulp. Arch. Path. 7: 215-227.

45. FANFESTI, B. E. 1967. The effect of temperature on zymogenic synthesis in the pancreas of the rat. Ph.D. Dissertation. Univ. of Michigan. Ann Arbor, Mich.

46. LEWIS, F. J. \& M. TAUFIC. 1953. Closure of atrial septal defects with the aid of hypothermia: Experimental accomplishments and report of a successful case. Surgery 33: 52-59.

Printed in the United States of A merica 